\title{
Pressure fluctuations from large-scale PIV over a serrated trailing edge
}

\author{
Lourenco T. Lima Pereira $^{1}\left[\right.$. Daniele Ragni ${ }^{1} \cdot$ Francesco Avallone $^{1} \cdot$ Fulvio Scarano $^{1}$
}

Received: 15 July 2019 / Revised: 9 January 2020 / Accepted: 14 January 2020 / Published online: 21 February 2020

(C) The Author(s) 2020

\begin{abstract}
Measurements of distributed surface pressure fluctuations over trailing-edge serrations at a Reynolds number $\operatorname{Re}_{\theta}=4900$ are performed with time-resolved 3D particle imaging velocimetry using helium filled soap bubbles as flow tracers. The sparse velocity vector field obtained with Lagrangian particle tracking is densely reconstructed using VIC+, a data assimilation technique based on the vortex-in-cell method. The instantaneous pressure distribution is inferred by invoking the momentum equation. Experiments are performed first over a flat plate, as assessment of the technique, where the properties of the convecting turbulent boundary layer are assessed and surface pressure fluctuations are validated against synchronous surface microphone measurements. The analysis of the flow over the trailing-edge serration focuses on the characterization of the spatial distribution and spectral coherence of surface pressure fluctuations, i.e. the flow features responsible for the acoustic scattering. Present results indicate that the measurement technique is suited to describe the spatio-temporal development of the pressure fluctuations over the serration surface at the proposed scale of the experiments.
\end{abstract}

\section{Graphic abstract}
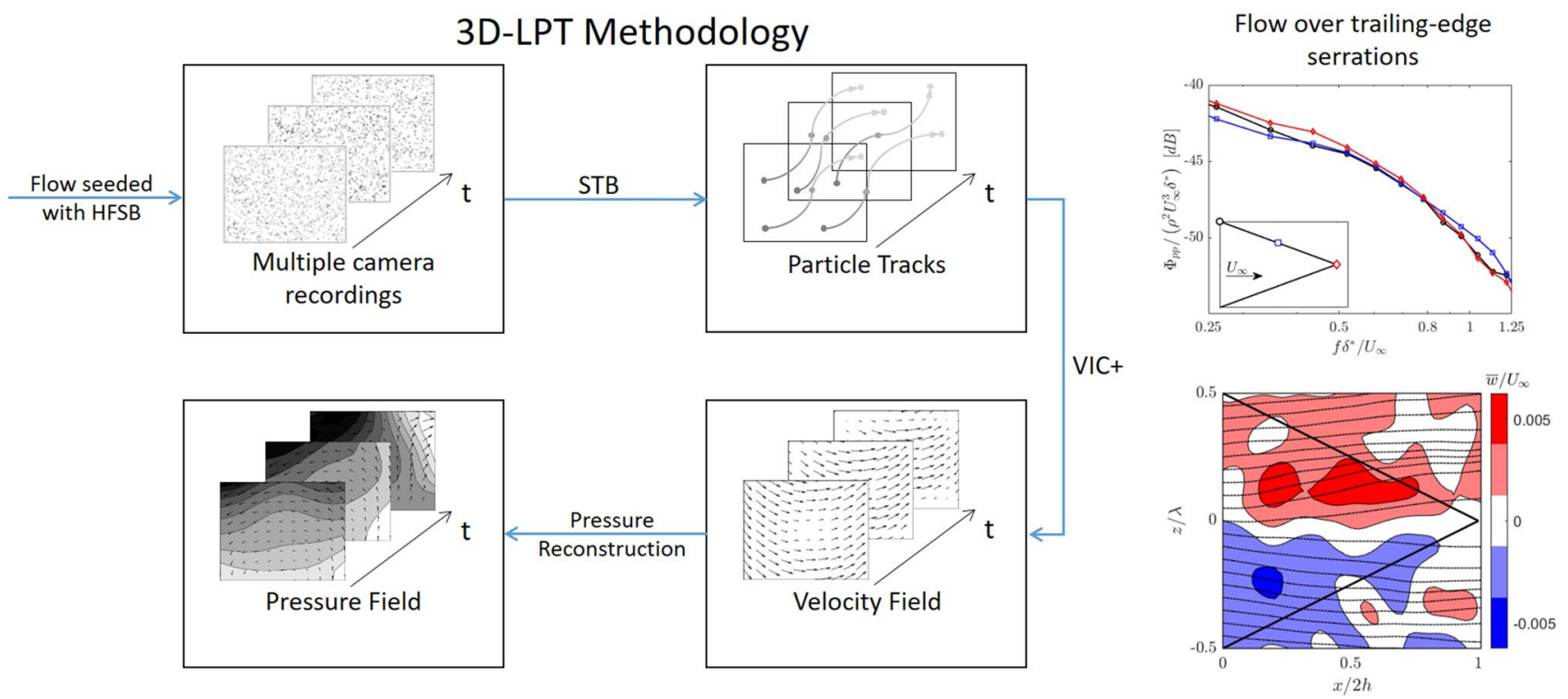

\section{Introduction}

The dataset of this research is available at https://doi.org/10.4121/ uuid:46879b06-bbf5-49ed-9c83-5d286c3371df.

Lourenco T. Lima Pereira

L.T.LimaPereira@tudelft.nl

1 Faculty of Aerospace Engineering, TU Delft, Kluyverweg 1, 2629HS Delft, The Netherlands
The dominant aerodynamic source of noise in wind turbines is turbulent boundary-layer trailing-edge noise TBLTE (Oerlemans et al. 2007). This is caused by the scattering of turbulent pressure fluctuations convecting across a sharp trailing edge, creating acoustic waves with intensity 
scaling with the 5th power of the free-stream Mach number (Howe 1978).

It has been proven that this noise source can be significantly mitigated by retrofitting the trailing edge with a sawtooth-like profile, known as trailing-edge serration (Howe 1991b). This noise reduction strategy mitigates the coherent scattering of the acoustic waves. However, the accurate modelling of the aerodynamic wavenumber-frequency spectrum and of the acoustic scattering, necessary for the far-field analytical models, is still an ongoing subject after the important works of Howe (1978, 1991a, b). Analytical predictions still depart from measurements, as reported by Gruber et al. (2011), although most recent analytical models proposed by Lyu et al. (2016) and Ayton (2018) seem to better account for the differences between predicted and measured far-field noise. Despite the above, significant discrepancies are still found (Sanjosé et al. 2019).

One of the main reasons for these discrepancies is that these models are based on the frozen turbulence assumption, i.e. the hypothesis that the boundary layer remains unaffected while convecting over the trailing edge. As a matter of fact, this approach uses the wavenumber spectrum model from Chase (1987) or, following the work of Roger and Moreau (2005), uses a semi-analytical model for the wall pressure spectrum beneath a turbulent boundary layer (Hwang et al. 2009) and the one of Corcos (Corcos 1964) for the spanwise correlation length. However, experimental work from Moreau and Doolan (2013) and Ragni et al. (2019) have shown that the frozen turbulence assumption does not hold over the serration as the statistical flow features (i.e., integral length scales and convective velocity) vary along the serrated trailing edge.

To support the development of more generally valid analytical models, a full characterization of the spatio-temporal behaviour of surface pressure fluctuations over trailing-edge serrations is needed. Numerical simulations have greatly contributed to their assessment, computing the unsteady pressure data over trailing-edge serrations (Jones and Sandberg 2012; Avallone et al. 2018) and assessing the analytical predictions for the far-field measured spectrum (Sanjosé et al. 2019). However, numerical simulations need support from detailed experimental investigations with high spatio-temporal requirements for benchmarking. To date, several experimental investigations have been performed with surface pressure transducers (Chong and Vathylakis 2015; Sanders et al. 2018) and time-resolved tomographic Particle Image Velocimetry (PIV) (Avallone et al. 2016). The former allows to locally measuring the surface pressure fluctuations, with a high temporal resolution, at the cost of perturbing the flow field when small thickness serrations are considered. On the other hand, tomographic PIV is non-intrusive and offers the advantage of spatially dense measurements, but the temporal resolution of the measurements is somewhat lower (typically up to $5 \mathrm{kHz}$ ).
For aeroacoustics purposes, measurements of surface pressure fluctuations and their spatial and temporal correlation can be retrieved from PIV data using high-speed three-dimensional time-resolved experiments, also termed 4D-PIV. The conventional analysis for PIV experiments is based on cross-correlation such as in planar and tomographic PIV (Raffel et al. 2018). Recent studies have shown that individual particle tracking over multiple exposures yields a more accurate estimation of velocity and acceleration. The latter type of analysis is referred to as Lagrangian Particle Tracking (LPT). The Shake-The-Box algorithm is a frequently used implementation of LPT data analysis as reported by (Schanz et al. 2016).

Following the work done by Ghaemi et al. (2012) and Pröbsting et al. (2015), spectra of surface pressure fluctuations obtained from PIV measurements were compared with reference surface pressure transducers, yielding a favourable agreement. A more recent study from Ragni et al. (2019) along a serrated trailing edge compared time-resolved tomographic PIV with Lattice-Boltzmann numerical simulations, indicating a good agreement also on the turbulent flow properties. However, the use of timeresolved tomographic PIV to perform surface pressure reconstruction remained restricted to measurement volumes in the order of $10 \mathrm{~cm}^{3}$ and turbulent flow regimes with Reynolds number $\operatorname{Re}_{\theta} \sim 2000$. Increasing further the Reynolds number is necessary to bring these techniques in the range of industrial applications $\left(\operatorname{Re}_{\theta} \sim 10,000\right.$ and with nonzero pressure gradient) and a significant upscaling step for the measurement volume is necessary.

The use of Helium-Filled Soap Bubbles (HFSB, Bosbach et al. 2009) and the introduction of accurate and efficient 3D Lagrangian Particle Tracking (3D-LPT, Schanz et al. 2016) for aerodynamics has partially overcome the spatial limitation, given the intense light scattering of HFSB tracers. 4D-PIV measurements were extended to domains in the order of $1000 \mathrm{~cm}^{3}$ (Scarano et al. 2015). Schanz et al. (2018) have demonstrated a significant upscale of the technique for wall turbulence studies in an atmospheric turbulent boundary layer, achieving meter range measurement volume.

The application of the technique for aeroacoustics requires solving a number of unknowns: the intrinsic spatial resolution of the measurements based on HFSB tracing and the required dynamic range of instantaneous velocity, vorticity and pressure field reconstruction. One of the fundamental steps to be clarified is the accuracy of the pressure fluctuations measurements from HFSB particle tracks. For this purpose, data assimilation of the sparsely distributed tracers is required, as it was demonstrated that conventional cross-correlation analysis would not achieve the requirements of spatial resolution. An assimilation technique, based on the vortex-in-cell method (VIC+, Schneiders and Scarano 2016), has proven the ability to resolve a wider range of scales for the velocity fluctuations. 
The pressure field reconstruction is then based on the solution of the Poisson problem as described in several studies from the literature (see the review from Van Oudheusden 2013).

Previous works, attempting to describe the spatio-temporal evolution of surface pressure fluctuations by means of 4D-PIV, have resulted in the compromise between spatial resolution, overall spatial range, and measurement accuracy. For instance, Pröbsting et al. (2013) reported a lower limit for the spanwise coherence of pressure fluctuations, based on both spatial resolution and the measurement intrinsic noise. It is not known whether the increased measurement dynamic range of LPT and the prospected reconstruction resolution of VIC+ data assimilation allow solving the above compromise when using more sparse HFSB tracers.

The present work explores the application of 4D-PIV to aeroacoustics using HFSB as tracers. For this purpose, the methodology is first assessed against the direct measurements of surface pressure transducers over a turbulent boundary layer. Afterward, the same method is applied to describe the spatio-temporal behaviour of the pressure fluctuations over trailing-edge serrations at $\operatorname{Re}_{\theta}=4900$. The focus is put on the role of the turbulent structures responsible for trailing-edge noise generation and the possible aerodynamic effects of the trailing-edge serrations over such structures. The article is structured as follows: a description of the wind tunnel facility, the wind tunnel model, measurement equipment, and data analysis methodology is given in Sect. 2; the validation of the technique using the measurements of the turbulent boundary layer developed over a flat plate is discussed in Sect. 3; the description of the measured flow over the serrated trailing edge and the analysis of the unsteady pressure field are reported in Sect. 4.

\section{Methodology}

\subsection{Wind tunnel, model and flow conditions}

Experiments are conducted in the low-speed open jet wind tunnel (W-Tunnel) of the Aerospace Engineering
Fig. 1 Top: general layout of experiment with wind-tunnel test section, wind tunnel model, laser illumination and 3D imaging system. Bottom: plate model geometry, with details of surface pressure instrumentation. Measurement domains in the turbulent boundary layer and at the trailing edge are highlighted in green. Details A and B reveal the serration geometry and the pinhole positioning, respectively. Dimensions are in $\mathrm{mm}$

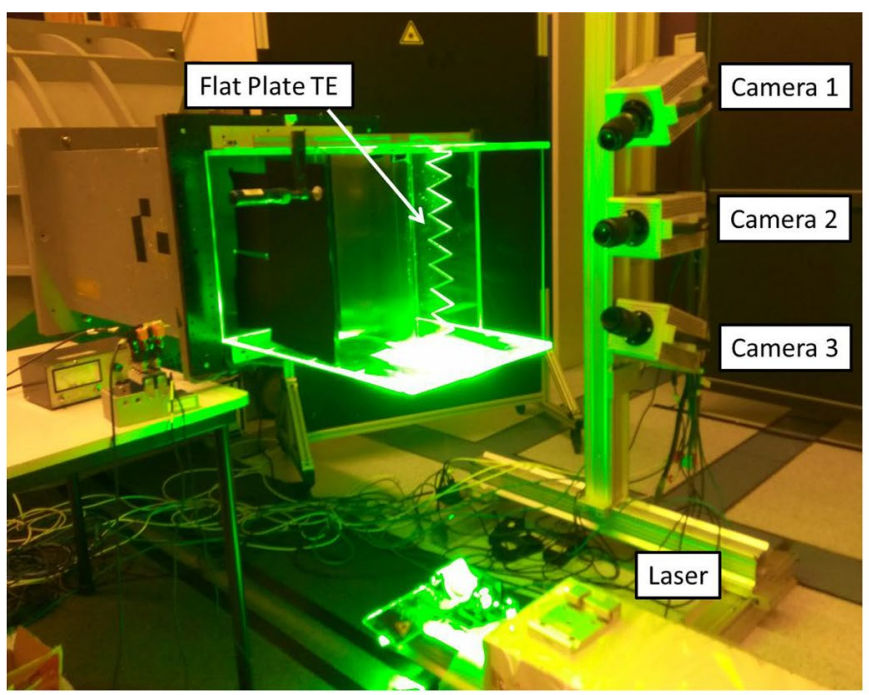

(a)

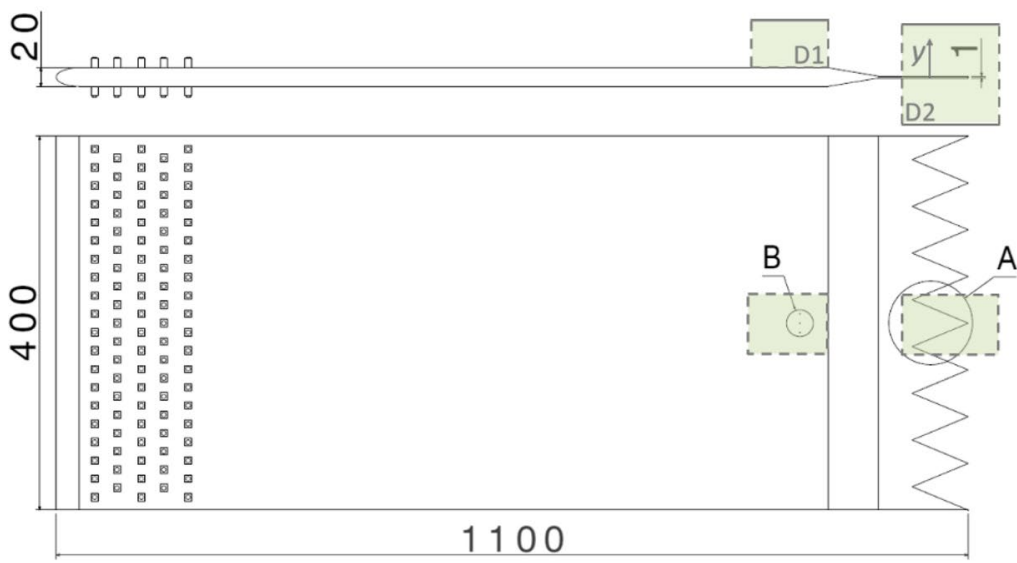

(b)

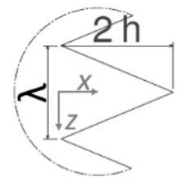

Detail A

A

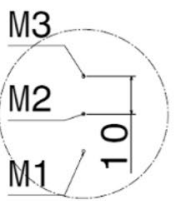

Detail B 
laboratories at TU Delft. The tunnel features a contraction ratio of 9:1 with a cross-section at the exit of $40 \times 40 \mathrm{~cm}^{2}$. Free-stream velocity is set to $10 \mathrm{~m} / \mathrm{s}$. The free-stream turbulence intensity is approximately $0.5 \%$.

A $1.1 \mathrm{~m}$ chord and $20 \mathrm{~mm}$ thick flat plate model is installed vertically along the entire span of the wind-tunnel test section (Fig. 1a). The plate thickness reduces smoothly to $1 \mathrm{~mm}$ at the trailing edge by an $8^{\circ}$ symmetrical wedge. The trailing edge is realized in transparent Plexiglas, allowing optical access through it. The serration is $60 \mathrm{~mm}$ long $(2 h)$ and $50 \mathrm{~mm}$ wide $(\lambda)$, resulting in a serration angle of $67^{\circ}(2 h / \lambda=1.2)$. This geometry (Fig. $\left.1 b\right)$ is representative of a trailing-edge serration for high Reynolds number applications.

Following Gruber et al. (2011), serrations offering a large noise reduction scale with the boundary-layer thickness. For the selected serration dimensions, the thickness of a turbulent boundary layer naturally transitioning and developing over a flat plate would be too small. For this reason, boundary-layer transition to turbulence is forced by employing five rows of obstacles with $10 \mathrm{~mm}$ height and $20 \mathrm{~mm}$ spacing in both the streamwise and spanwise directions. The first row of turbulators is placed $30 \mathrm{~mm}$ from the plate leading edge to generate a fully turbulent boundary layer at the measurement region. This arrangement follows the work of Rodríguez-López et al. (2016) who demonstrated that a thicker boundary layer can be obtained with no significant alterations to its aerodynamic properties.

Measurements are taken at two locations: a first region located $800 \mathrm{~mm}$ downstream of the leading edge (D1 in Fig. 1b), while a second region encompasses the serrated trailing edge (D2 in Fig. 1b). Measurements in the first region are conducted to validate the measurement technique and to infer the resolvable scales of the reconstructed surface pressure. In this region, 4D-PIV data are compared with pitot measurements and wall-mounted microphone transducers, following a similar procedure as reported by Pröbsting et al. (2013). Measurements in the second region are carried out to quantify surface pressure fluctuations over serrations; data are analysed following the approach suggested by Avallone et al. (2016).

\subsection{Measurement apparatus}

The HFSB seeder consists of 200 bubble generators installed on 10 rakes aligned vertically and covering a cross-section of $1 \mathrm{~m}$ height and $0.5 \mathrm{~m}$ width. The seeder is placed in the wind-tunnel settling chamber, past the turbulence screens; the seeded stream-tube contracts to approximately $40 \times 20$ $\mathrm{cm}^{2}$ in the test section. The HFSB tracers are, within $10 \%$ deviation, neutrally buoyant and have a diameter of approximately $400 \mu \mathrm{m}$. The estimated time response of the tracers is about $20 \mu$ s (Scarano et al. 2015). The measured tracer concentration is illustrated in Fig. 2. Approximately, 6-8 tracers $/ \mathrm{cm}^{3}$ are present in the boundary layer above the plate. The concentration drops rapidly approaching the wall to less than $2 \mathrm{~mm}$ distance, similar to what was reported by Faleiros et al. (2018). The number of particles detected on the opposite side of the plate is significantly lower upstream of the trailing edge, due to the limited optical transmission across the Plexiglas interface. Past the edge, tracers are detected in a higher number, although the concentration upstream of the plate remains not symmetrical as the seeded stream was positioned to favour the measurements above the plate.

Illumination is provided by a Quantronix Darwin Duo $\mathrm{Nd}$ :YLF laser $(2 \times 25 \mathrm{~mJ}$ at $1 \mathrm{kHz})$. The laser light propagates tangentially to the plate surface and is reflected back by a plane mirror to enhance uniformity of illumination and

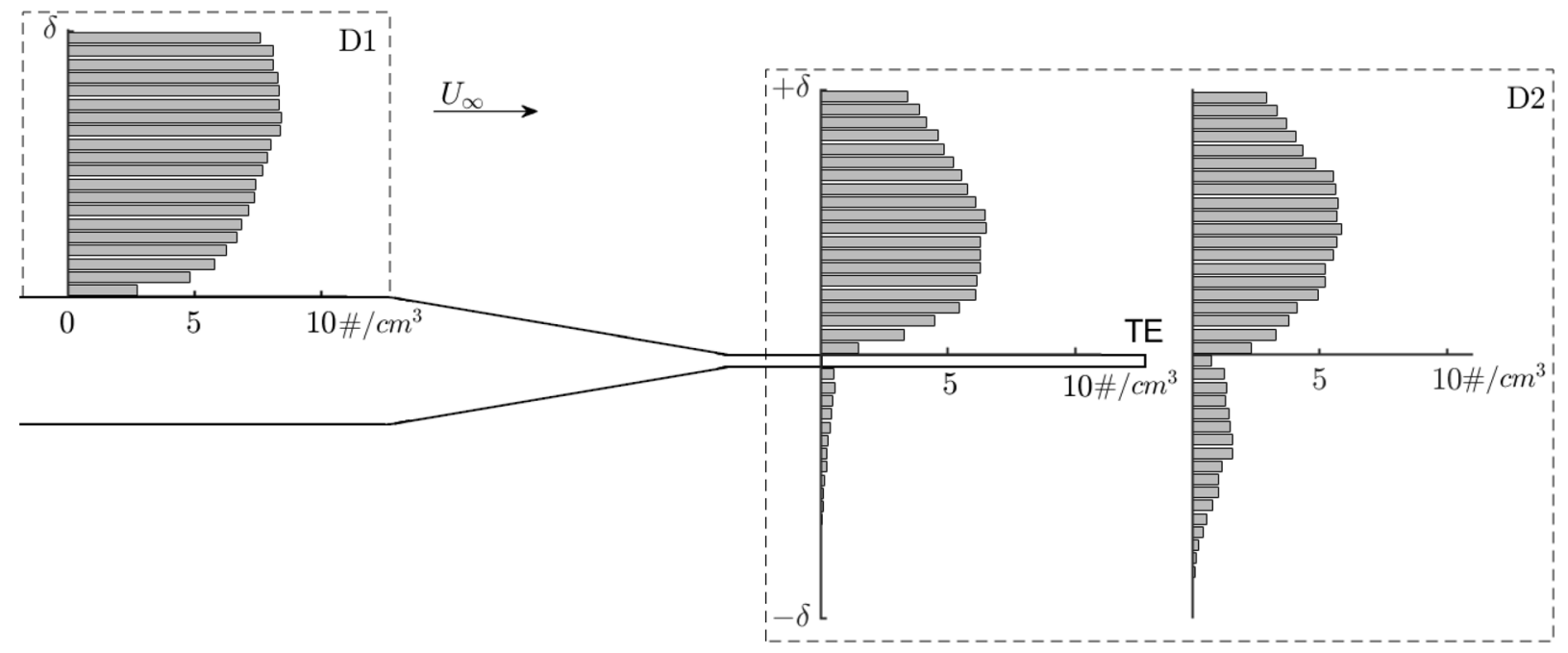

Fig. 2 Illustrative view of the spatial concentration of detected particle tracks for the turbulent boundary-layer measurement (left D1) and the trailing-edge serration measurement upstream and downstream from the trailing edge (D2) 
reduce shadow regions close to the surface. Three CMOS cameras (Photron FastCam SA1, $1 \mathrm{Mpx}$ at $5400 \mathrm{fps}$ ) subtend an arc of approximately $40^{\circ}$ (see Fig. 1a). The cameras are equipped with Nikon objectives of $60 \mathrm{~mm}$ focal length at aperture $f_{\#}=16$. The field of view is approximately $10 \times 10$ $\mathrm{cm}^{2}$, yielding a three-dimensional measurement domain of $9 \times 6 \times 5 \mathrm{~cm}^{3}$ and $10 \times 10 \times 7 \mathrm{~cm}^{3}$ for the boundary layer (D1) and trailing edge (D2), respectively. The image-to-object calibration is performed with a target plate and residual errors are corrected to within $0.1 \mathrm{px}$ applying the 3D selfcalibration algorithm (Wieneke 2008). Illumination and imaging are synchronized with a LaVision programmable timing unit (PTU 9) controlled with the DaVis 8 software.

Three Sonion $8010 T$ microphones are installed below the wall through a pinhole arrangement to provide direct measurement of the surface pressure fluctuations. The pinhole diameter is $1 \mathrm{~mm}$, while the cavity below is $0.2 \mathrm{~mm}$ high and has a diameter of $2.6 \mathrm{~mm}$, resulting in a Helmholtz resonance frequency of $4.6 \mathrm{kHz}$. The microphones are spaced by $10 \mathrm{~mm}$ along the span to measure the spanwise correlation length (M1, 2 and 3 in Fig. 1b). Calibration is performed by synchronously recording a white noise played by a far-field speaker with the Sonion microphones and a reference LinearX M51 microphone. In turn, the LinearX transducer is previously calibrated with a G.R.A.S. 42AC pistonphone. The sensitivity is estimated by comparing the RMS values of the installed microphones to the reference one, both band-pass filtered from 100 to $4000 \mathrm{~Hz}$ (flat response region of the Sonion microphones). Acquisition is carried out with a NI 9234 board with 24 bits ADCs and built-in anti-aliasing and AC filters. Synchronous microphone measurements are taken with a sampling frequency of $25,600 \mathrm{~Hz}$ for $10 \mathrm{~s}$. Camera clock and triggering signals are recorded along with the microphones for the time alignment of the time series.

\subsection{D-PIV data processing}

Several steps are necessary to obtain the three-dimensional velocity and pressure fields from the particle images. The motion of the particles is recorded in time-resolved mode and the image sequence is analysed to infer the trajectories of the HFSB tracers. Subsequently, the dense velocity field is reconstructed and finally, the instantaneous pressure field is evaluated and compared with the wall-mounted sensors. The process is schematically illustrated in Fig. 3 along with the methodologies used at each step, which are further described in the remainder.

\subsubsection{Lagrangian particle tracking}

The LPT technique is performed using the STB algorithm (Schanz et al. 2016) implemented in DaVis 8. Tracks with a minimum number of 9 exposures are considered as valid. The particle concentration and the main parameters of the measurement and data processing are summarized in Table 1. Based on the results of Schneiders and Scarano (2016), a concentration of 500 particles $/ \delta^{3}$ is sufficient to accurately capture the turbulent velocity fluctuations in the boundary layer. The analysed data are taken from the upper side of the wind tunnel model $(y>0)$, where the particle concentration is more homogeneously distributed. The lower side is only used in the near wake (Fig. 2).

\subsubsection{Dense velocity field reconstruction}

The velocity field is obtained from the discrete particle tracks using the VIC+algorithm (Schneiders and Scarano 2016). It is based on the minimization of the cost function $J=J_{u}+\alpha^{2} J_{D u}$ for the solution of the vorticity equation
Fig. 3 Schematic flow chart for the data processing of the raw recordings with LPT and reconstruction of dense velocity field with data assimilation. The analysis is concluded with instantaneous pressure estimation

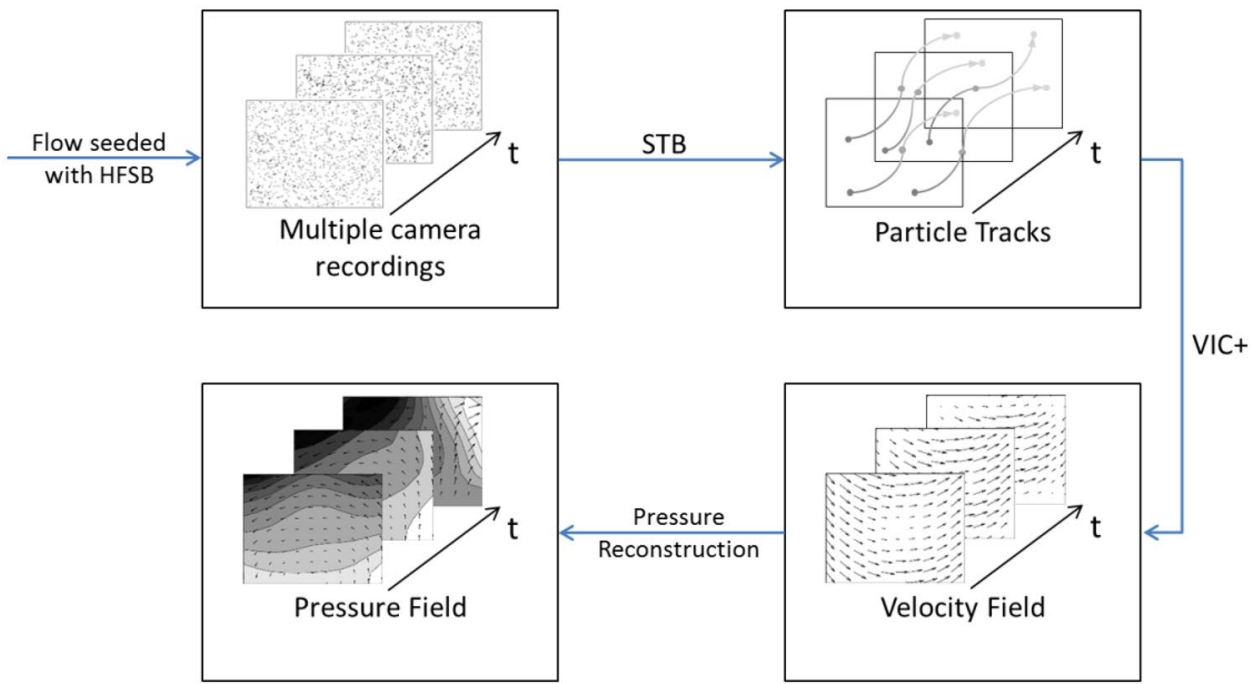


Table 1 Velocity field measurement parameters

\begin{tabular}{llll}
\hline & Units & Boundary layer & Trailing edge \\
\hline Recording rate & $\mathrm{Hz}$ & 5400 & 5400 \\
Average imaging resolution & $\mathrm{px} / \mathrm{mm}$ & 10.4 & 9.3 \\
& $\mathrm{px} / \delta$ & 520 & 460 \\
Number of recordings & & 5400 & 5400 \\
Measurement volume & $\mathrm{cm}^{3}$ & $9 \times 6 \times 5(270)$ & $10 \times 10 \times 7(700)$ \\
Particle concentration & $\delta^{3}$ & $1.8 \times 1.2 \times 1.0$ & $2.0 \times 2.0 \times 1.4$ \\
& $\# / \mathrm{cm}^{3}$ & 7.2 & 4.5 \\
Velocity vector grid spacing & $\# / \delta^{3}$ & 900 & 560 \\
& $\mathrm{~mm}$ & 1.5 & 1.5 \\
& $\delta$ & 0.03 & 0.03 \\
\hline
\end{tabular}

in a gridded data domain. $J_{u}$ and $J_{D u}$ are, respectively, the residual sum of squares (RSS) of the velocity and velocity material derivative errors estimated at the particle location.

The VIC+ reconstruction yields data on a Cartesian grid with a spacing of $\Delta x=1.5 \mathrm{~mm}$ (Table 1). The spacing is based on the grid discretization criterion of $1 / 4 C^{-1 / 3}$, where $C$ is the mean particle concentration. At the solid wall, no-slip boundary condition is imposed for the boundarylayer case. In the wake of the trailing edge, no boundary condition is imposed.

The reconstructed data close to the outer domain boundaries (edge of the boundary layer and in- and out-flow surfaces) suffers from increased oscillations caused by the lower particle concentration. The latter typically decays within five grid points towards the interior of the computational domain. The data domain used for pressure evaluation is, therefore, a sub-domain with respect to that analysed with VIC+. The criterion for cropping is based on the analysis of the divergence error (mass conservation principle). The components of the velocity divergence in a scatter plot are shown in Fig. 4, where the term with larger uncertainty $(\partial u / \partial x)$ is presented on the horizontal axis. The data dispersion decreases rapidly as one moves from the frontier of the domain towards its interior. Based on the data, the domain volume is cropped by approximately $10 \%$ at its borders to avoid the spurious oscillations in the pressure reconstruction scheme, as also suggested in Schneiders and Scarano (2016). Data in the selected sub-domain fall inside the blue (turbulent boundary layer) and red (trailing-edge serrations) areas in the graphs, where the error is below $0.002 \%$ of the overall mass flow within the measurement region.

With this PIV technique, the maximum resolvable frequency of the velocity fluctuations that can be resolved is given by Eq. (1). The equation relates the frequency with the convective mean velocity $\left(u_{\mathrm{c}}\right)$ and the minimum resolvable length scale $\left(\lambda_{\min }\right)$. The latter is estimated both with the inter-particle distance $\left(C^{-1 / 3}\right)$ and with the correlation length of the Gaussian radial basis function interpolator used $\left(\lambda_{\min }=\sqrt{2 \pi} a \Delta x\right)$, where $a=1.1$. Resulting

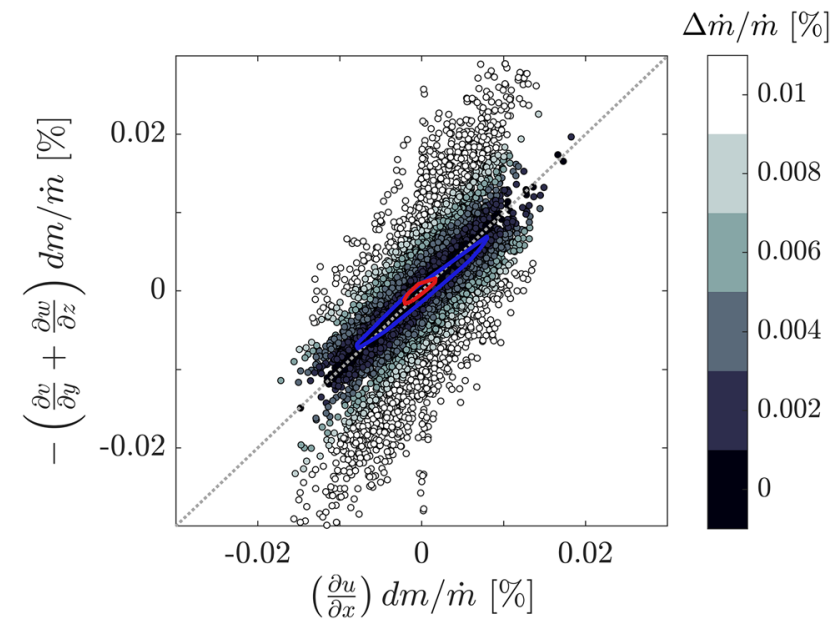

Fig. 4 Mass flow disparity (distance from the dotted diagonal) obtained from VIC+ for the turbulent boundary layer. Results after domain cropping are represented within the blue (turbulent boundary layer) and red (trailing-edge serrations) areas

cut-off frequencies are estimated in $f_{\mathrm{LPT}}=1300 \mathrm{~Hz}$ and $f_{\mathrm{VIC}}=1900 \mathrm{~Hz}$, respectively.

$f_{\max }=\frac{u_{\mathrm{c}}}{\lambda_{\min }}$.

\subsubsection{Pressure from PIV and related parameters}

Pressure fluctuations are obtained by the reconstruction technique introduced by Gurka et al. (1999). The integration procedure follows more closely the work of Ghaemi et al. (2012): the Poisson Eq. (2), in which the viscous term is neglected, is solved numerically by an implicit second-order finite difference method using a lower-upper (LU) factorization to solve the sparse linear system. 
$\nabla^{2} P=-\rho \nabla \cdot \frac{\mathrm{D} \vec{V}}{\mathrm{D} t}$

Neumann boundary conditions are applied to in-flow, out-flow and lateral boundaries as well as at the solid wall. Dirichlet condition, based on Bernoulli equation with the instantaneous velocity field (Eq. (3)), is applied at the edge of the boundary layer. Boundary surfaces where Neuman and Dirichlet boundary conditions apply are denoted by dashed and solid lines in Fig. 1b, respectively. In the equation, $p_{\infty}$ and $U_{\infty}$ refer to the free-stream pressure and velocity. The source term is obtained directly from the velocity material derivative. The particle trajectory is modelled through a polynomial regression of order 2 in a centred stencil of 5 time steps $(n=2)$. The values chosen demonstrated convergence for the pressure spectrum measured.

$p=p_{\infty}+\frac{1}{2} \rho\left(U_{\infty}^{2}-\vec{V} \cdot \vec{V}\right)$.

The correlation length $\left(l_{z}\right)$ and the convection velocity $\left(u_{\mathrm{c}}\right)$ are relevant quantities for the analysis of the sound source (Amiet 1976). Both are derived from the pressure field. The first is calculated in Eq. (4), where $\gamma\left(f, z^{\prime}\right)$ is the coherence function between the reference position and the point separated in spanwise direction by $z^{\prime}$ at a given frequency $f$. A Gaussian curve fit $\left(\gamma^{2}\left(z^{\prime}\right)=\mathrm{e}^{\left(z^{\prime} / l\right)^{2}}\right)$ of the measured coherence curve with $z^{\prime}$ is used to estimate the spanwise correlation length. Based on Eq. (4) and the fitting parameter $l, l_{z}$ is given by $\sqrt{\pi / 2} l$.

$l_{z}=\int_{0}^{\infty} \gamma\left(f, z^{\prime}\right) \mathrm{d} z^{\prime}$

The estimation of the convection velocity is carried out following Romano (1995), where the variation in frequency of the phase shift $(\phi)$ along the streamwise direction is used, Eq. (5). The derivative in the equation is estimated with a linear fit from the cross-spectrum phase, obtained from the Welch's method:

$u_{\mathrm{c}}=2 \pi \Delta x{\frac{\mathrm{d} \phi^{-1}}{\mathrm{~d} f}}^{-1}$

To avoid the influence of the spatial stencil size on the estimation of the correlation length and the wavenumberfrequency spectra along the serration, a stencil of only 8 points $(12 \mathrm{~mm})$ is used, corresponding to less than $20 \%$ of the serration width.

The analysis of the turbulent structures most relevant to the pressure fluctuations is attempted by performing conditional averaging, following the work of Ghaemi and Scarano (2013). Averages are obtained for a condition of minimum pressure, following Eq. (6), where $\hat{C}$ denotes the conditional average and $\langle C\rangle$ the averaging operator of a function $C$.

$\hat{C}=\langle C\rangle \mid p(\vec{x})>p_{\text {rms }}$.

\subsubsection{Analysis of experimental uncertainties}

The uncertainty in the velocimetry measurements with 3D-LPT is due to particle displacement estimation error. This error, for the case of HFSB, is given by the distance between the two glare points $\left(d_{\mathrm{g}}\right)$ within the same bubble, estimated as $d_{\mathrm{g}}=d_{\mathrm{b}} / \sqrt{2}$, where $d_{\mathrm{b}}$ is the bubble diameter. The uncertainty in the measurement of particle location affects the estimation of the displacement. The regularization of the particle tracks reduces the displacement uncertainty depending on the chosen stencil size $(N)$, as suggested in Lynch and Scarano (2013). Accordingly, the resulting random error in the measurement of the instantaneous velocity $\varepsilon_{\mathrm{u}}$ is calculated as in Eq. (7). For an average bubble diameter of $400 \mu \mathrm{m}$, considering an average stencil size of 11 time steps and a displacement random error $\left(\varepsilon_{x}\right)$ equal to $d_{\mathrm{g}}$, the resulting random error in the velocity fluctuations is $0.04 \mathrm{~m} / \mathrm{s}\left(0.4 \% U_{\infty}\right)$.

$\varepsilon_{\mathrm{u}}=\frac{\varepsilon_{x}}{\Delta t} N^{-3 / 2}$.

Uncertainty in the reconstructed instantaneous pressure field depends on the error made in the estimation of the source term, i.e. the truncation of the Lagrangian derivatives $\varepsilon_{\mathrm{Du}, \text { trunc }}$ and the random errors in the measurement of the material derivative $\varepsilon_{\text {Du,PIV }}$ (Violato et al. 2011; Ghaemi et al. 2012). The uncertainty in the estimation of the pressure $\varepsilon_{\mathrm{p}}$ can be evaluated using the linear error propagation procedure according to Eq. (8) (Ragni et al. 2019). The latter does not consider errors from the integration procedure, considered here minor in comparison to the Lagrangian derivative estimation and the velocity uncertainty.

$\varepsilon_{\mathrm{p}} \approx \rho h\left(\varepsilon_{\mathrm{Du}, \mathrm{trunc}}+\varepsilon_{\mathrm{Du}, \mathrm{PIV}}\right) \approx \rho \Delta x\left(\frac{1}{2}(n \Delta t)^{2}\left|\frac{\mathrm{D} \vec{V}}{\mathrm{D} t}\right| \frac{|\nabla \cdot \vec{V}|}{\Delta t}+\frac{\varepsilon_{\mathrm{u}}}{2 n \Delta t}\right)$.

From the experimental data, the truncation error is approximately $44 \mathrm{~m} / \mathrm{s}^{2}$, and the error in the estimation of the material derivative is $56 \mathrm{~m} / \mathrm{s}^{2}$. This yields an uncertainty in the instantaneous pressure of $0.3 \mathrm{~Pa}(0.5 \%$ of the flow dynamic pressure).

Table 2 compares the uncertainties from the current experiment with other measurements performed with tomographic PIV. As expected, $\varepsilon_{\mathrm{u}}$ is significantly reduced with 
Table 2 Comparison of measurement uncertainties between the current experiment and similar works

\begin{tabular}{lllll}
\hline & $\begin{array}{l}\text { Current } \\
\text { experiment } \\
\text { (TBL) }\end{array}$ & Ghaemi et al. (2012) & $\begin{array}{l}\text { Pröbsting } \\
\text { et al. (2015) }\end{array}$ & Ragni et al. (2019) \\
\hline Measurement volume & $270 \mathrm{~cm}^{3}$ & $5.6 \mathrm{~cm}^{3}$ & $3.6 \mathrm{~cm}^{3}$ & $11.5 \mathrm{~cm}^{3}$ \\
Flow speed & $10 \mathrm{~m} / \mathrm{s}$ & $9.3 \mathrm{~m} / \mathrm{s}$ & $10 \mathrm{~m} / \mathrm{s}$ & $20 \mathrm{~m} / \mathrm{s}$ \\
$\varepsilon_{\mathrm{u}}$ & $0.04 \mathrm{~m} / \mathrm{s}$ & $0.02 \mathrm{~m} / \mathrm{s}$ & $0.07 \mathrm{~m} / \mathrm{s}$ & $0.1 \mathrm{~m} / \mathrm{s}$ \\
$\varepsilon_{\mathrm{p}}$ & $0.3 \mathrm{~Pa}$ & $0.05 \mathrm{~Pa}$ & $0.14 \mathrm{~Pa}$ & $0.08 \mathrm{~Pa}$ \\
\hline
\end{tabular}

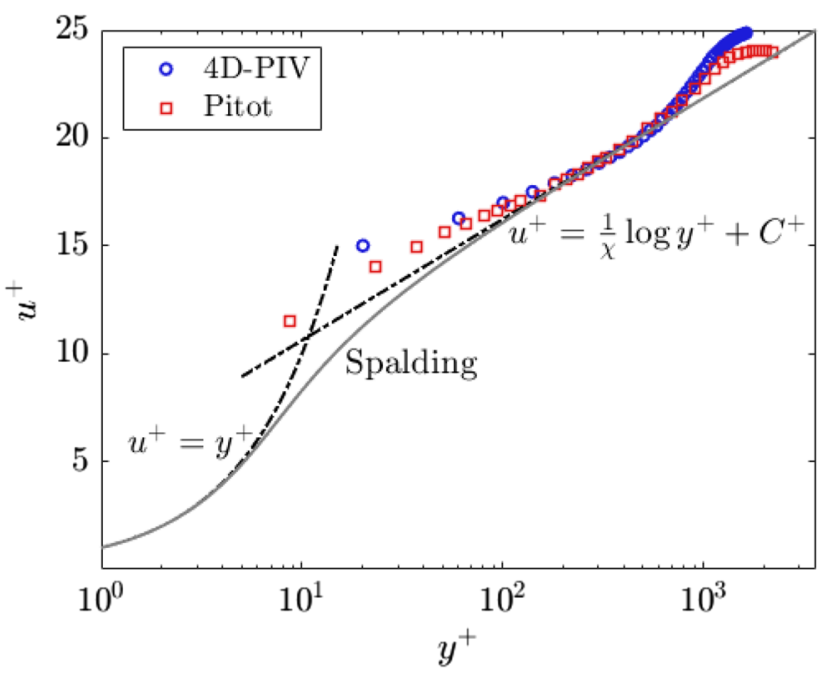

(a)

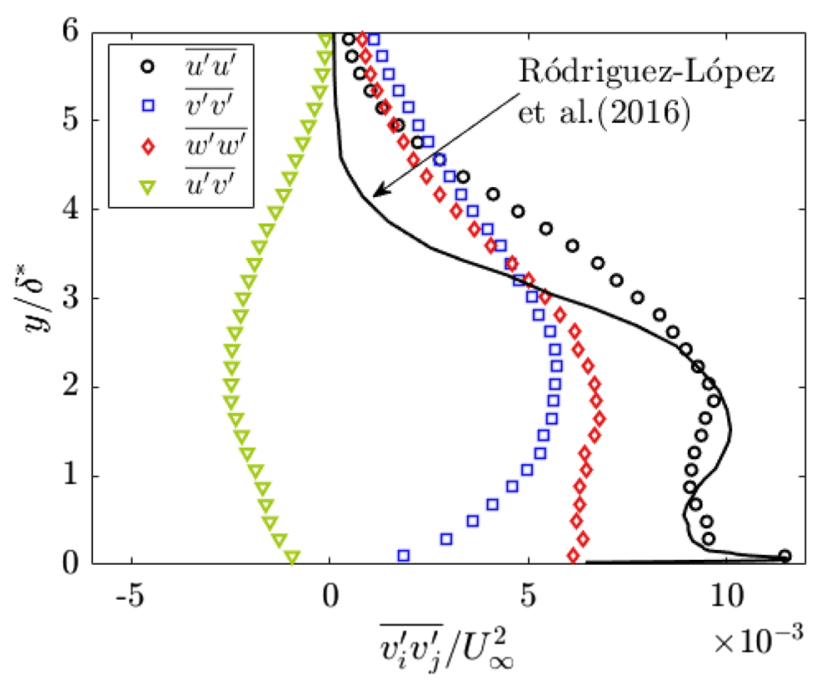

(b)

Fig. 5 Comparison between 4D-PIV and Pitot mean velocity measurements (left). Wall-normal Reynolds stresses profiles from 4D-PIV (right)

the LPT approach even at a lower image resolution. Conversely, $\varepsilon_{\mathrm{p}}$ is larger because of the dependency of the integration scheme on the grid spacing in Eq. (8).

\section{Turbulent boundary-layer measurements}

Measurements of the statistical properties of the turbulent boundary layer over the flat plate are carried out for assessing a-posteriori the fidelity of the measurement technique. Velocimetry results obtained from 4D-PIV are plotted against pitot probe measurements and compared with the law-of-the-wall model (Fig. 5a). The Spalding and logarithmic fit are obtained using the constants $\chi=0.41$ and $C^{+}=5$, following the procedure proposed in Clauser (1956). Pitot data are corrected following Bailey et al. (2013). Results show a good agreement for the mean boundary layer with some departures at the lower and upper boundaries. The latter may be ascribed to the lower particle concentration in the measurements. The characteristic shape factor $\mathrm{H}=1.4$ and the fitting shown in Fig. 5(a) indicate a fully developed turbulent boundary layer with zero pressure gradient.

Figure 5b depicts the Reynolds stresses measured with 4D-PIV. Values are in agreement with those reported by
Table 3 Boundary-layer quantities obtained from 4D-PIV measurements

\begin{tabular}{lll}
\hline Parameter & Symbol & Value \\
\hline Boundary-layer thickness & $\delta_{99}$ & $56 \pm 1 \mathrm{~mm}$ \\
Displacement thickness & $\delta^{*}$ & $10.3 \pm 0.2 \mathrm{~mm}$ \\
Momentum thickness & $\theta$ & $7.4 \pm 0.2 \mathrm{~mm}$ \\
Friction velocity & $u_{\tau}$ & $0.39 \pm 0.01^{\mathrm{a}} \mathrm{m} / \mathrm{s}$ \\
Shape factor & $H$ & 1.39 \\
Reynolds & $\operatorname{Re}_{x}$ & 650,000 \\
& $\operatorname{Re}_{\delta}$ & 33,200 \\
& $\operatorname{Re}_{\delta^{*}}$ & 6800 \\
& $\operatorname{Re}_{\theta}$ & 4900 \\
\hline
\end{tabular}

${ }^{a}$ Estimated from the fitting of the logarithmic region from the boundary layer

Rodríguez-López et al. (2016) under similar Reynolds conditions. Table 3 shows the boundary-layer integral parameters obtained from the measurements. $\delta_{99}$ is estimated from the boundary-layer profile and is used as limit for the integral boundary-layer parameters, performed with a trapezoidal rule integration. Uncertainties are estimated based on the 4D-PIV technique errors. Friction velocity uncertainty is given by the quality of the logarithmic fit. 
The measurement of turbulent pressure fluctuations and their spatio-temporal correlation is initially assessed comparing the time history of the surface pressure fluctuations with the wall-mounted reference microphones.

A statistically relevant comparison is made using the cross-correlation coefficient as well as the frequency spectrum. The pressure time history is illustrated in Fig. 6a for the centre microphone (M2), where a fair agreement is found between the two datasets. Correlation between the three microphones and the 4D-PIV measurements (Fig. 6c) is maximum at null time delay and is estimated in about 0.6. Similar correlation values were found in previous investigation where pressure time signals reconstructed from PIV were compared with surface pressure data (Ghaemi et al. 2012; Pröbsting et al. 2013). Disagreements in the time series are attributed to three factors: the acoustic noise from a non-acoustically treated facility; the experimental uncertainty of both measurement techniques; and the extrapolation of the pressure calculated within the flow field to the microphone location. The latter is especially important in the present context, where HFSB tracers are significantly sparser near the wall.

Spectra obtained from the two datasets (Fig. 6b) show good agreement in the range of Strouhal numbers based on the displacement thickness between $0.2 \leq \mathrm{St}_{\delta^{*}} \leq 1.0$ (frequency range between 200 and $1000 \mathrm{~Hz}$ ), i.e. within the universal $\left(0.2<\mathrm{St}_{\delta^{*}}<0.5\right)$ and inner scale ranges $\left(\mathrm{St}_{\delta^{*}}>0.5\right)$ (Hwang et al. 2009). At higher frequencies, the PIV-based pressure spectrum underestimates the amplitude of the fluctuations, indicating that the smaller structures

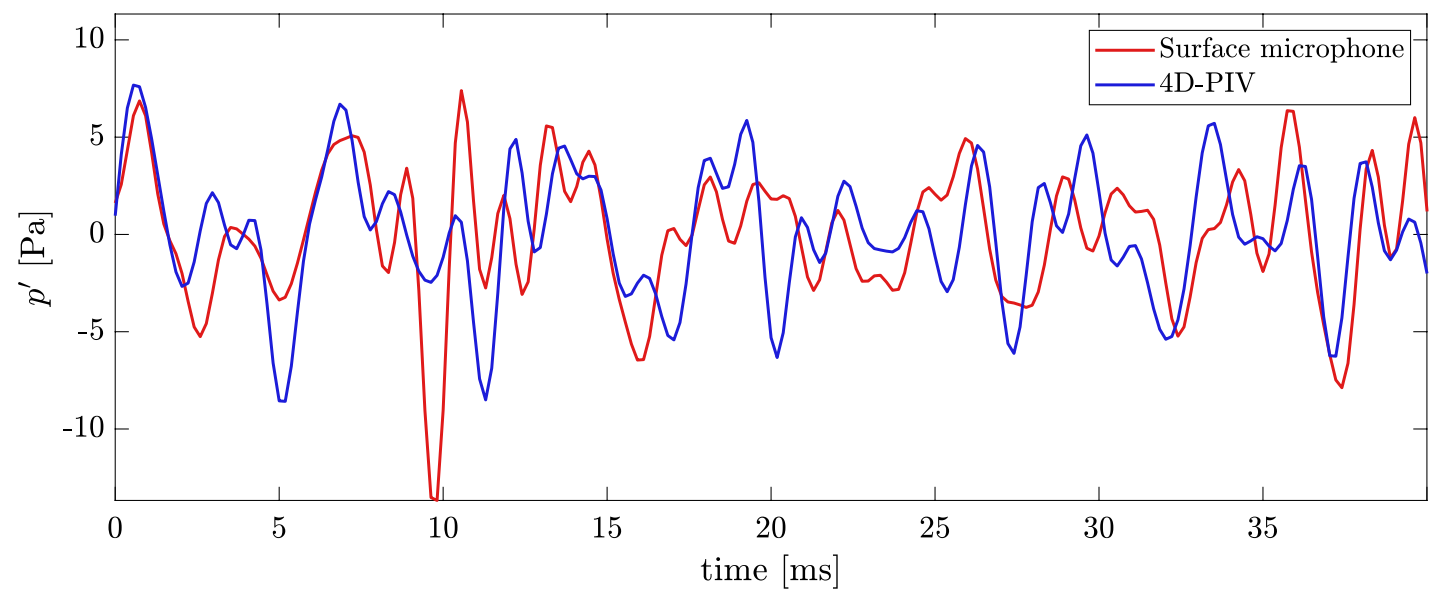

(a)

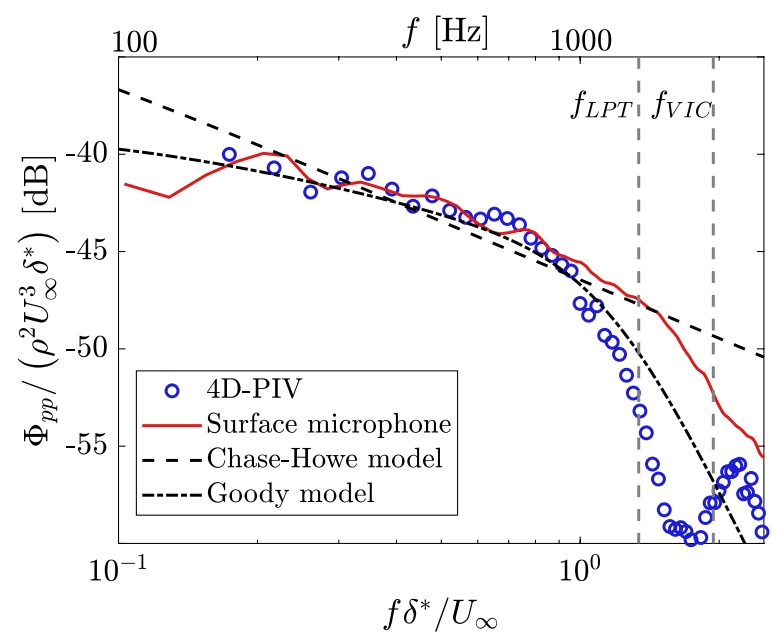

(b)

Fig. 6 Reconstructed pressure from 4D-PIV and comparison with microphone measurements. a Pressure time series, b pressure spectrum (Chase-Howe and Goody models are given in dashed lines), and

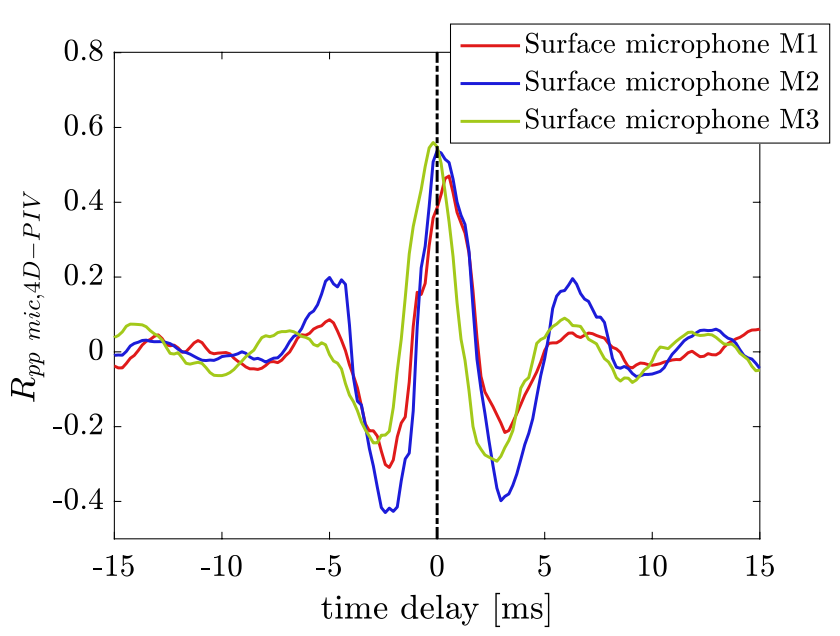

(c)

c PIV-microphone correlation $\left(R_{\mathrm{pp}}\right)$ as a function of the time delay between the two measurements 


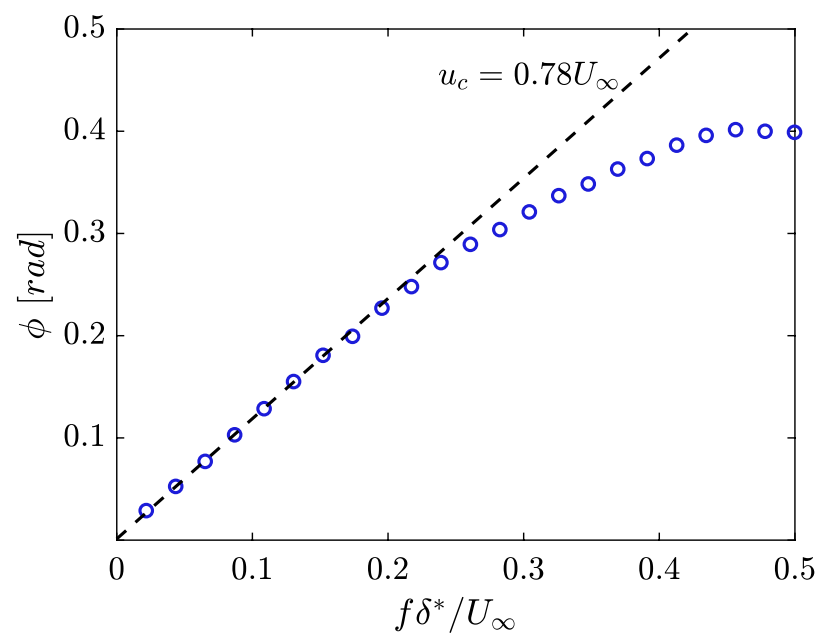

(a)

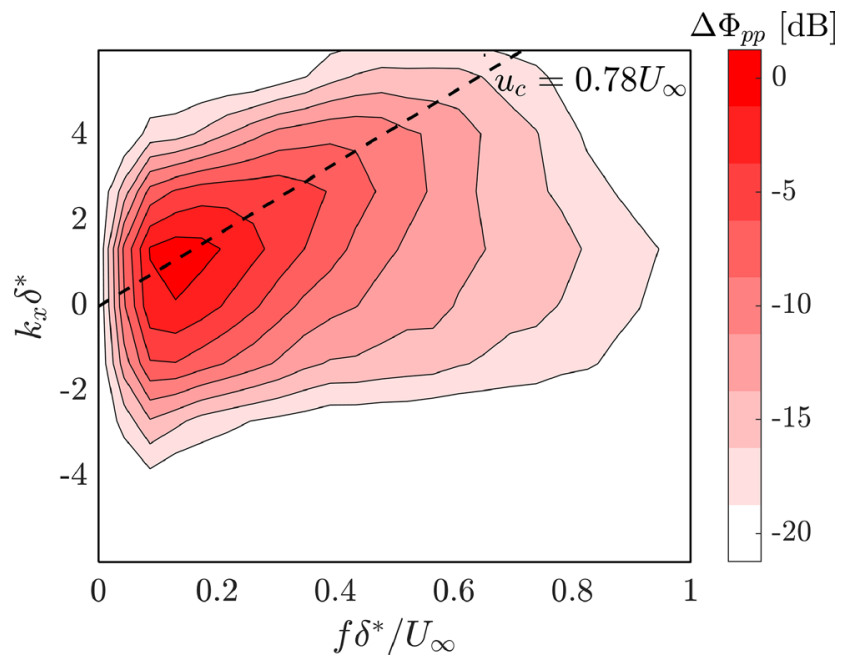

(b)

Fig. 7 Convection velocity estimated from phase shift along two consecutive points in streamwise (a) and wavenumber-frequency spectrum of the pressure fluctuations (b)

$\left(l_{z}<C^{-1 / 3}\right)$ are not well captured with the PIV technique. For this reason, the following analyses are restricted at frequencies smaller than $f_{\mathrm{LPT}}$. In the same figure, the pressure spectra from two analytical models (Chase-Howe model; Howe 1998, and Goody model; Goody 2008) are compared with the experimental data. An agreement is observed for $\mathrm{St}_{\delta^{*}}<1.0$, which is expected since the Chase-Howe model is valid in the universal range and does not account for the effect of viscous terms introduced by smaller structures. The model from Goody (2008) improves the predictions to the inner scales, with the agreement maintained up to the limit given by $f_{\mathrm{LPT}}$.

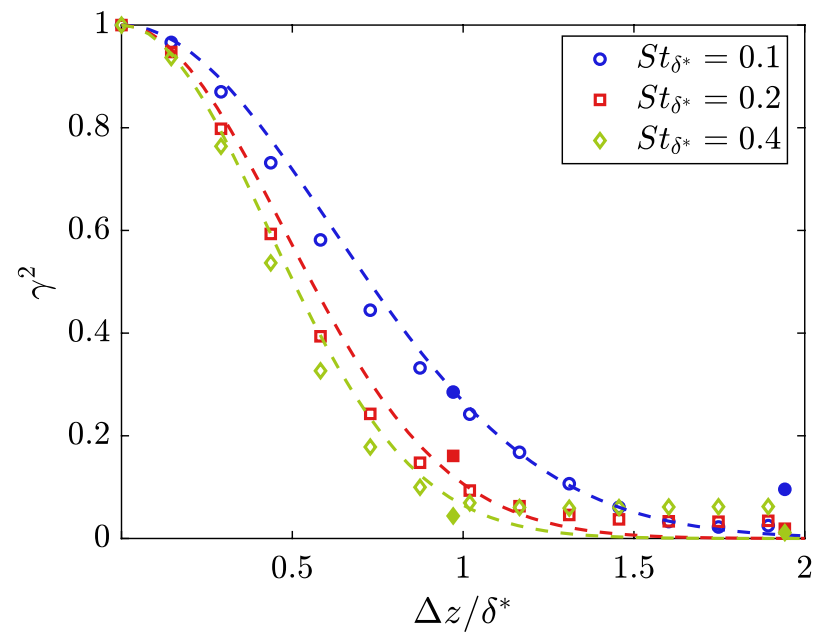

(a)

Fig. 8 Spanwise coherence of the surface pressure fluctuations (a). Filled symbols represent microphone measurements, while open symbols represent measurement from 4D-PIV; the dashed curves are the
The convective velocity, estimated using Eq. (5), is $0.78 U_{\infty}$ (Fig. 7a). Noise in high frequency range along with the smaller size of the eddies affect the fit, thus creating a phase loss in the measurements for $\mathrm{St}_{\delta^{*}}>0.25$. This is also observed at the wavenumber-frequency spectrum (Fig. 7b), where the curve connecting the local maxima, i.e. the convective ridge, becomes horizontal at high frequency, indicating the contamination of the data by the noise.

Figure 8 a compares the spanwise coherence obtained from pressure fluctuations measured with surface microphones (filled symbols) and from the 4D-PIV (open symbols). A good agreement is found between the two, with

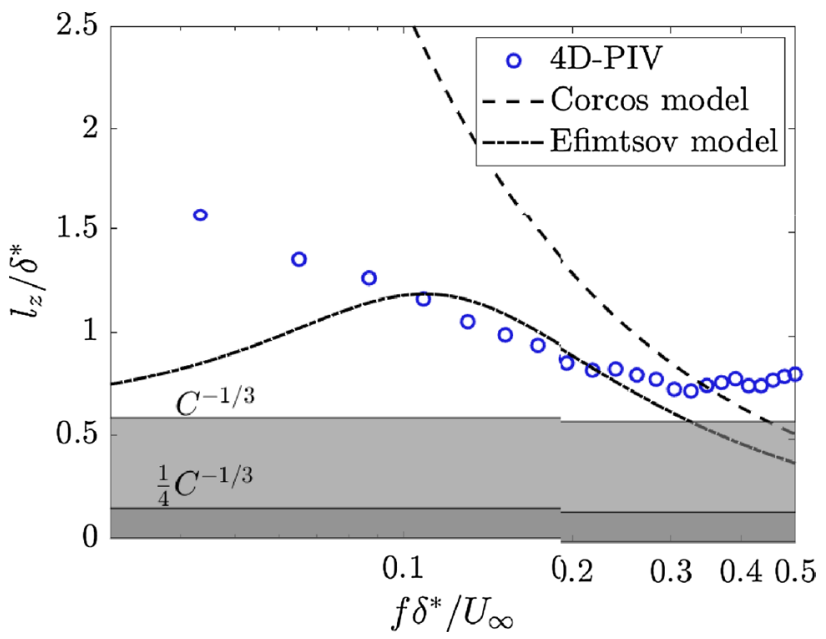

(b)

Gaussian fit from the 4D-PIV data. Comparison between estimated correlation length from 4D-PIV and models from Corcos and Efimtsov (b) 
higher deviation in the low coherence region, where the sensitivity to noise is higher. In Fig. 8b, the correlation length from Eq. (4) is compared with the Corcos and Efimtsov models, with the latter based on the values from Palumbo (2012). The results are similar to the ones of Pröbsting et al. (2015) with similar discrepancies between the analytical models and the measurements. Similar to the previous study, the correlation length can be measured up to $\mathrm{St}_{\delta^{*}}=0.4$. This limit is set by the mean inter-particle distance $\left(C^{-1 / 3}\right)$.

An instantaneous flow visualization of the velocity field through iso-surface of the Q-criterion for vortex identification is demonstrated in Fig. 9a, b. In the same figure, slices representing the instantaneous pressure field are also presented. In the picture, high-pressure fluctuations are seen underneath the hairpin head $(\mathrm{H})$, similarly to what was found by Ghaemi and Scarano (2013). The sweep (S) and the ejection (E) regions are represented by iso-surfaces in blue and red, respectively. Conditional averaging performed using a positive fluctuation of amplitude equal to the maximum root mean square (Fig. 9c, d) highlights the same structures observed in the single time step shown. The conditional position on the high side of the inner boundary-layer region is used to enforce the large structures only. Underneath the hairpin head, high-pressure fluctuations are again observed. Alongside, low-pressure is seen to be induced downstream from the hairpin location, similar to the observations from Ghaemi and Scarano (2013). The latter also pointed to the presence of low-pressure fluctuations at the hairpin legs, as also observed in Fig. 9c.

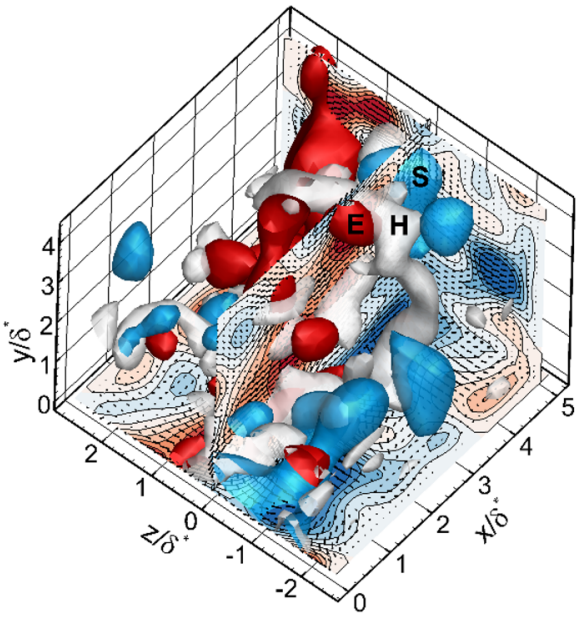

(a)

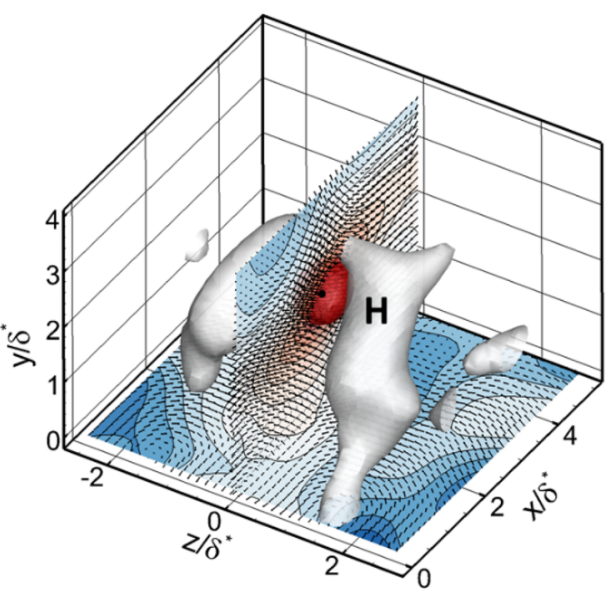

(c)

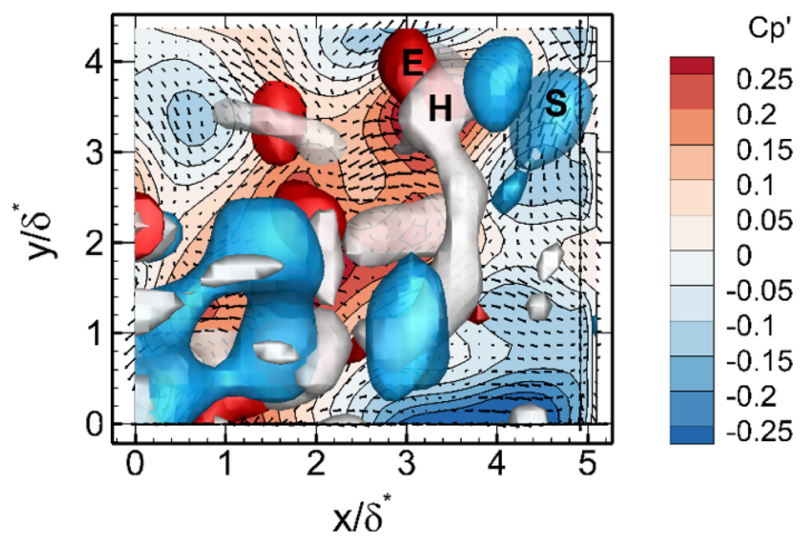

(b)

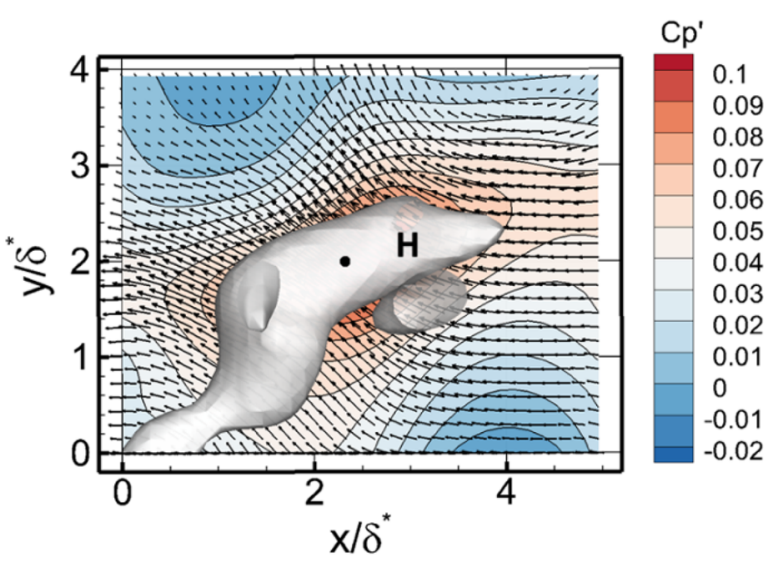

(d)
Fig. 9 Instantaneous flow field at the turbulent boundary layer (a). Iso-surfaces of the wall-normal velocity fluctuations $\left(v^{\prime}= \pm 0.12 U_{\infty}\right)$ are represented in red and blue and of the $Q$-criterion $(Q=0.09)$ in grey. b Shows a side view of the same structures. c, d Depicts the conditional averaging of high positive pressure fluctuations at the location represented in black 


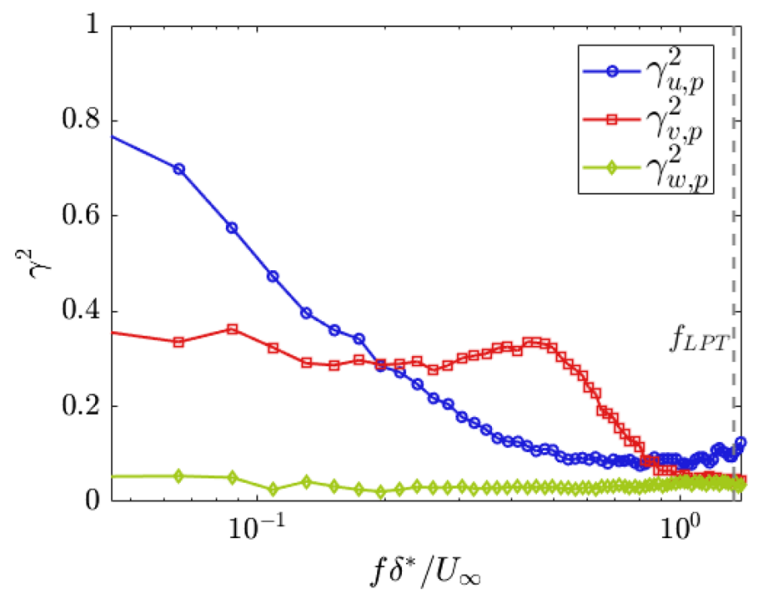

Fig. 10 Coherence calculated between pressure fluctuations and all the three velocity components measured close to the wall

The coherence between pressure and each of the velocity components close to the wall is shown in Fig. 10b. The diagram depicts two different Strouhal number regions. For $\mathrm{St}_{\delta^{*}}<0.2$, pressure fluctuations are seen to correlate better with the streamwise velocity. On the other hand, fluctuations in the universal range $\left(0.2<\mathrm{St}_{\delta^{*}}<0.5\right)$ follow the wall-normal velocity component, as also found by Johansson et al. (2006).

This section has shown that the LPT with HSFB is able to capture the spatio-temporal correlation of the surface pressure fluctuations within the predicted frequency limit. In the following, the approach is extended to a more complex flow field, i.e. the flow over a trailing-edge serration.

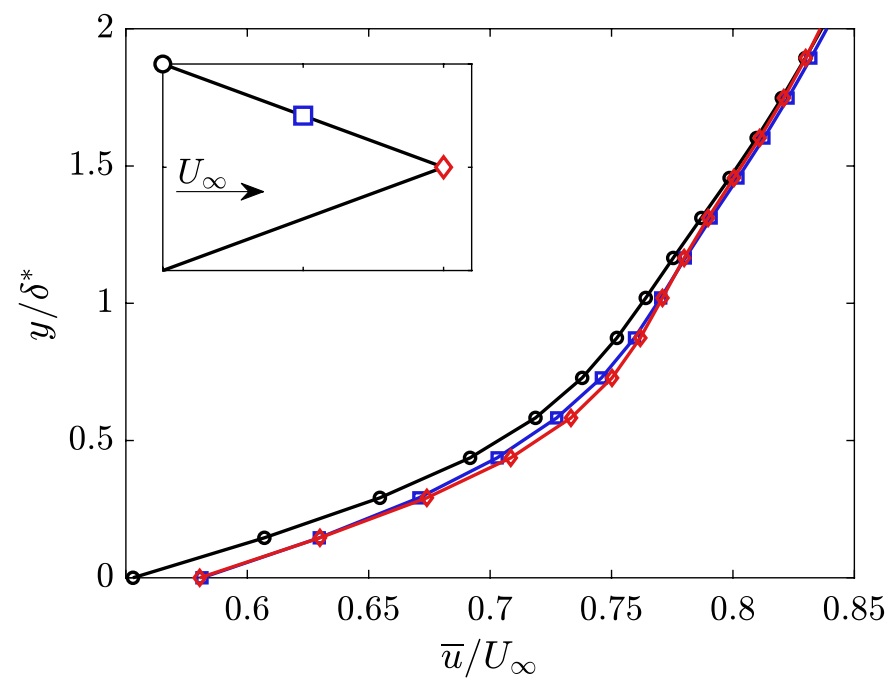

(a)

\section{Trailing-edge serration measurements}

The same procedure adopted in Sect. 3 is used for the analysis of the flow over the serrated trailing edge. Figure $11 \mathrm{com}-$ pares the mean and Reynolds stresses profiles taken along the serration edge with detail in the region of higher velocity fluctuations $\left(y / \delta^{*} \leq 2\right)$.

The mean streamwise flow velocity, calculated using free slip condition with VIC+, shows a slight increase in the velocity close to the wall in the most downstream direction. The Reynolds stresses along the edge (Fig. 11b) reduce in the streamwise direction particularly close to the wall. The development of the flow in the wake reduces the high shear observed near the wall, consequently bringing $\overline{u^{\prime} u^{\prime}}$ values closer to the levels in the outer layer. Wall-normal shear $\left(\overline{v^{\prime} v^{\prime}}\right)$ and the $\overline{u^{\prime} v^{\prime}}$ component also reduce in the along the edge, similar to what was found by Avallone et al. (2018).

Detailed insights into the flow features induced by the trailing-edge serrations are given by the mean flow contours in the $x-z$ plane at $y / \delta^{*}=2$ (Fig. 12). The flow over the serrations is slightly slower along the serration surface with respect to the wake region between teeth (Fig. 12a). Even at zero-degree angle of attack, the flow tends to seep into the empty space between serrations (downward motion) as evidenced by the negative mean wall-normal velocity component near the edge shown in Fig. 12b. As a consequence, the flow exhibits an outward motion, visible from the spanwise velocity component shown in Fig. 12c and from the mean flow direction in the $x-z$ plane (Fig. 12d). This effect is attributed to the three-dimensional shear layer that promotes the formation of streamwise elongated structures in the empty space between serrations. The flow features

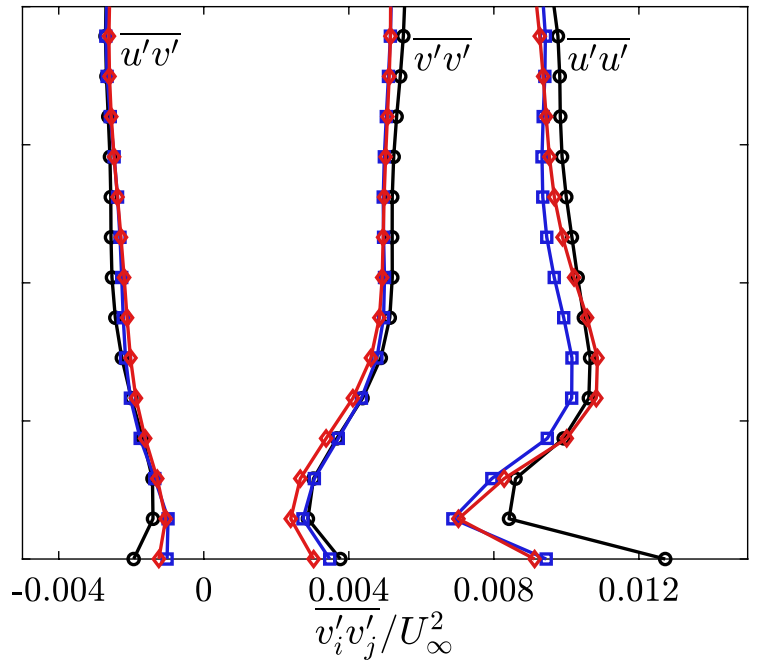

(b)

Fig. 11 Mean velocity and Reynolds stresses measured along the serration edge at $x / 2 h=0,0.5$ and 1.0. a Mean streamwise velocity; $\mathbf{b}$ mean velocity in spanwise direction; c Reynolds stresses $\left(\overline{u^{\prime} u^{\prime}}, \overline{v^{\prime} v^{\prime}}\right.$ and $\left.\overline{u^{\prime} v^{\prime}}\right)$ 


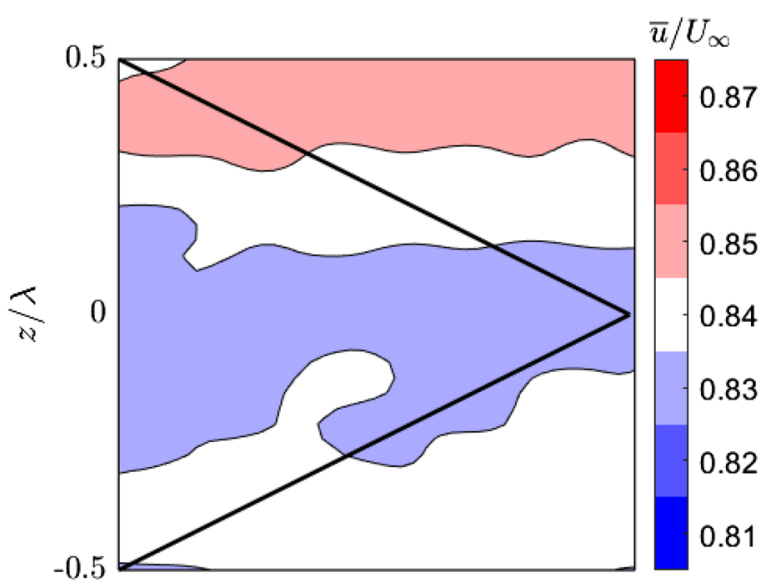

(a)

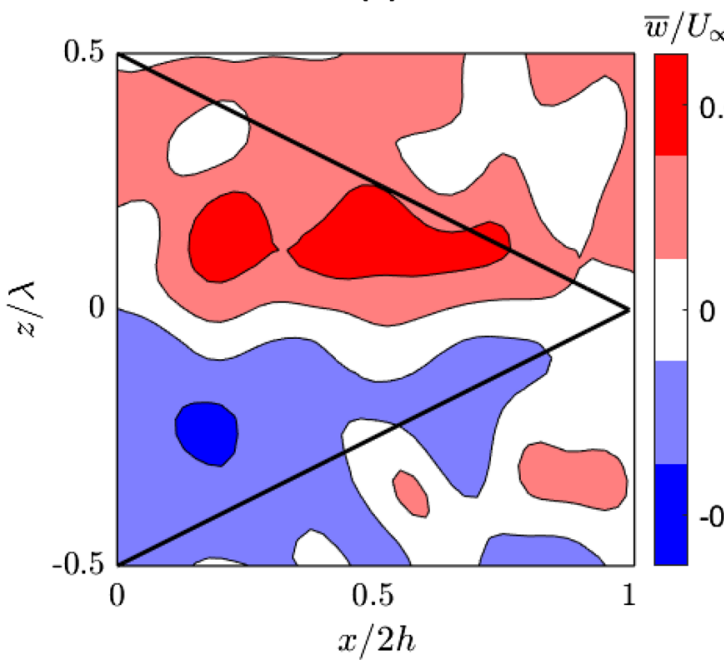

(c)

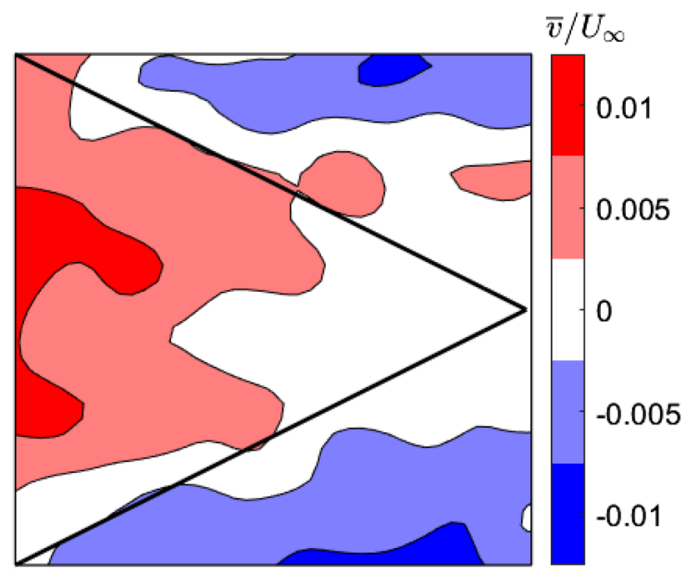

(b)

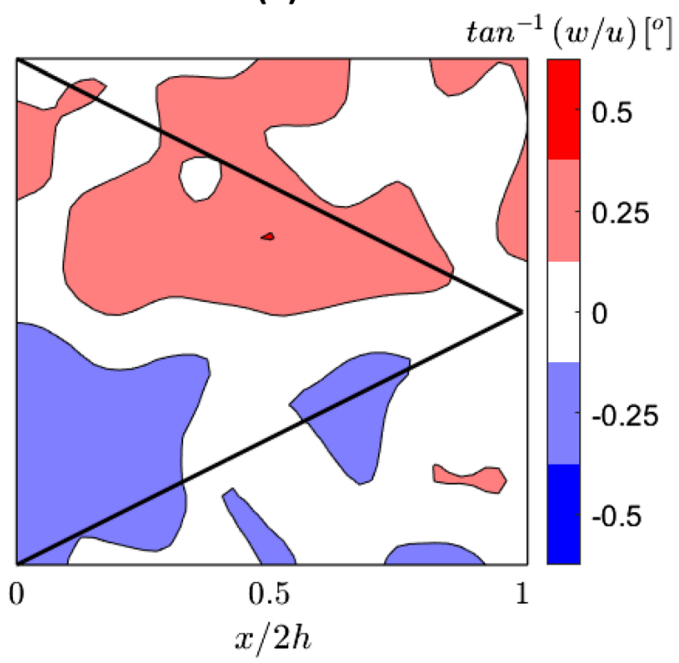

(d)

Fig. 12 Mean flow velocity contours along the trailing-edge serration: a streamwise velocity; $\mathbf{b}$ wall-normal velocity; $\mathbf{c}$ spanwise velocity and $\mathbf{d}$ spanwise flow angle

detected in this experiment are very similar to the ones found in previous studies and they confirm that the technique captures the relevant physics induced by the presence of the serrations.

After having assessed that the relevant flow physics is well captured, the pressure field can be studied. The reconstructed pressure spectra at three positions along the serration edge are shown in Fig. 13a. Similar to the velocity fluctuations, the pressure is seen to be almost unaltered along the trailing-edge serration. Figure $13 \mathrm{~b}$ reveals a minor increase of the pressure fluctuations amplitude at higher frequencies at the centre of the serration $(0.2<x / 2 h<0.8)$ and at mid-frequencies at its tip $(x / 2 h=1.0)$. Fluctuations are only decreased at very low frequencies $\left(\mathrm{St}_{\delta^{*}}<0.4\right)$ in the center of the serration. The differences between spectra are more clearly shown in Fig. 14 where $\Delta \phi_{\mathrm{pp}}$, the difference between the spectra at a given streamwise location along the edge and the one at $x / 2 h=0$, is plotted. This clearly shows that the major differences are found in the range $0.2<x / 2 h<0.8$, i.e. the region where the downward and sideward motions are dominant. The findings indicate how the effectiveness of the trailing-edge add-ons is influenced by the wake development along the trailingedge gap, in opposite to the frozen turbulence assumption. Increased levels of the large structures will affect the performance of the trailing-edge serration close to the tip of the serration while small structures will degrade the performance along the centre of the serration. Gruber et al. (2011) also observed a decrease in the velocity fluctuations at low frequencies and an increase at higher frequencies along the wake region. Chong and Vathylakis (2015) also pointed to higher fluctuations along the tip of the serrations at lower frequencies and around the centre of the serration for higher frequencies. 


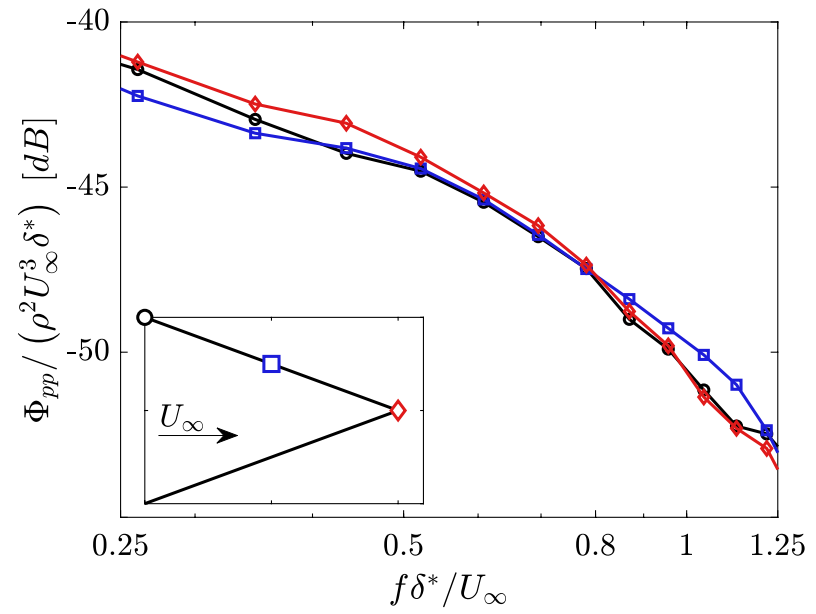

(a)

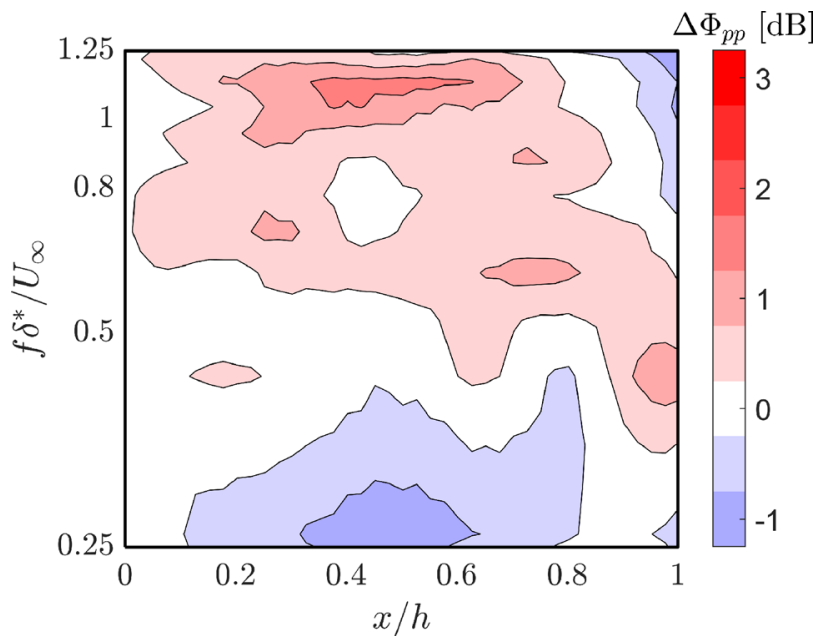

(b)

Fig. 13 Pressure spectrum over the serration edge (a) and variation of the pressure spectrum in relation to the spectrum at the root (b)

Fig. 14 Third-octave band pressure spectrum contour $\left(\Delta \Phi_{\mathrm{pp}}=\Phi_{p p}-\Phi_{\mathrm{pp}}^{x=0, z=0}\right)$ along the serration surface

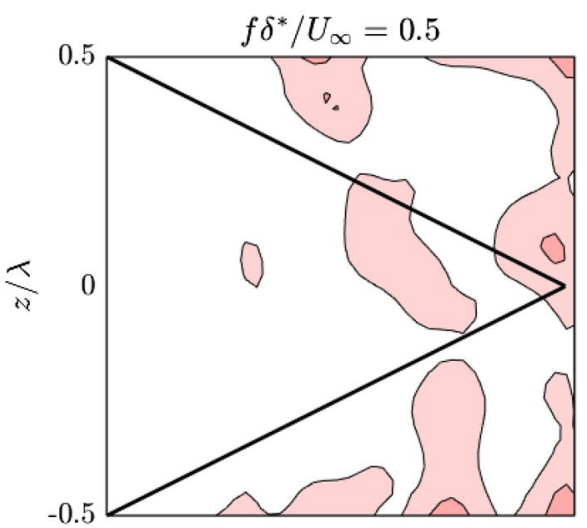

(a)

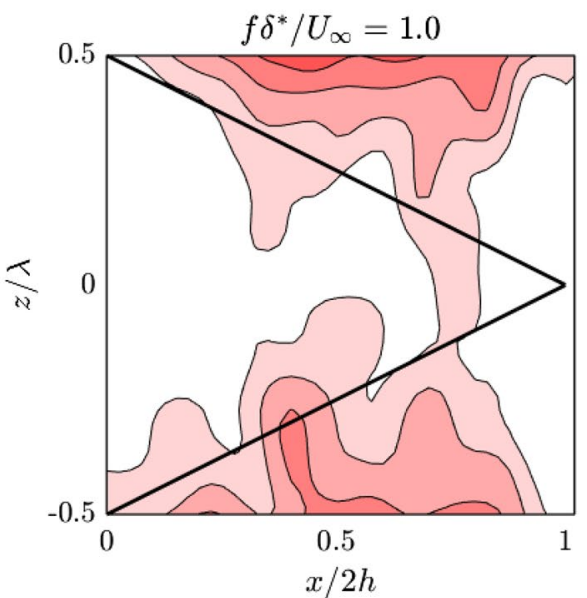

(c)

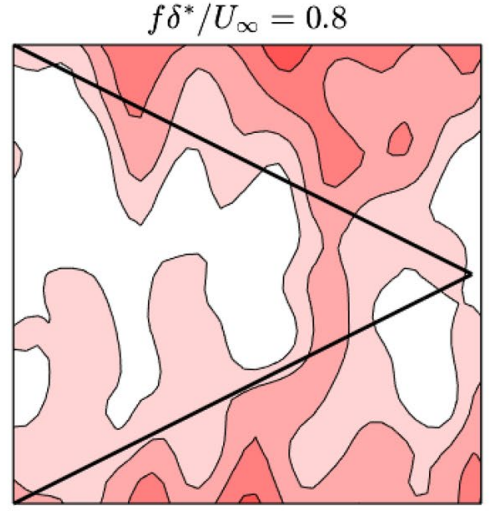

(b)

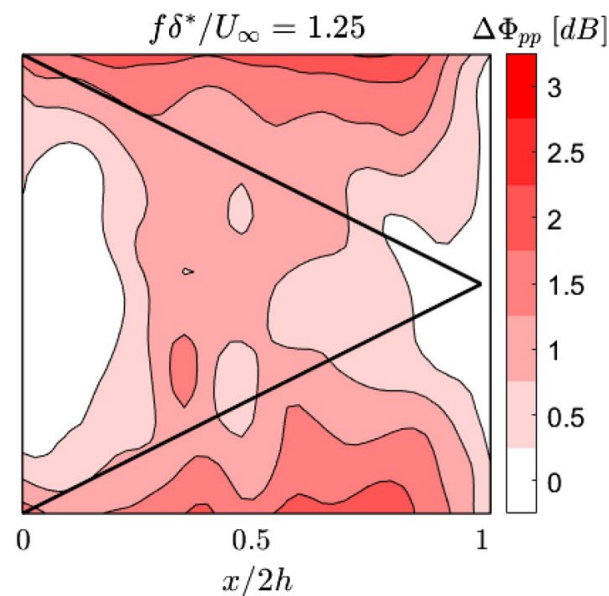

(d) 


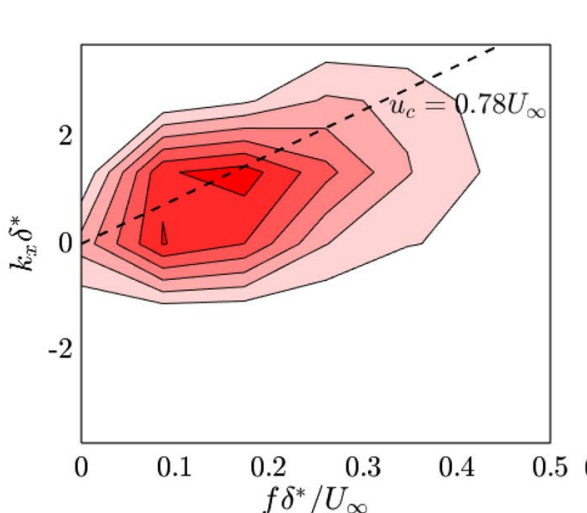

(a)

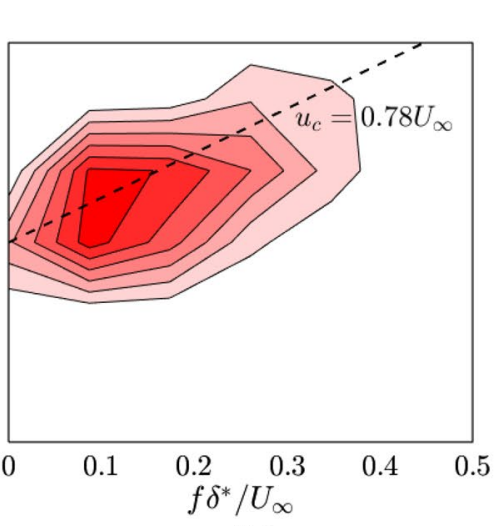

(b)

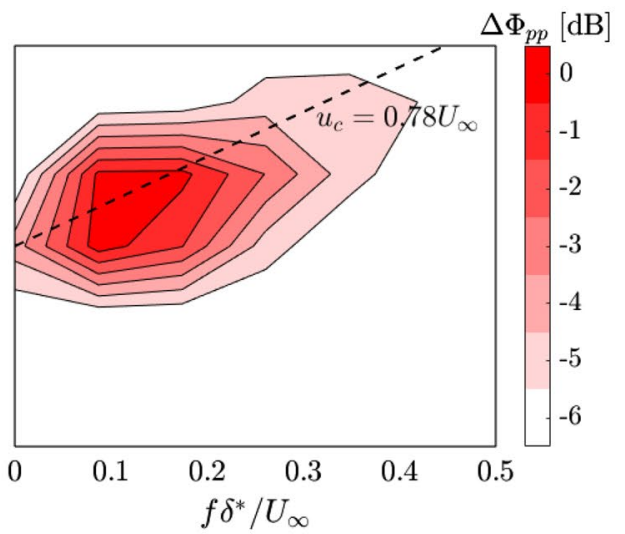

(c)

Fig. 15 Wavenumber frequency spectrum along the serration edge. a $x / 2 h=0, \mathbf{b} x / 2 h=0.5, \mathbf{c} x / 2 h=1.0$

Figure 15 shows the predicted wavenumber-frequency spectrum along the serration edge. This indicates the strong similarities with the measurements obtained for the boundary layer over the flat plate (Fig. 7b). An absolute maximum is measured at $\mathrm{St}_{\delta^{*}}=0.1$ and the convection velocity is $0.78 U_{\infty}$. For Strouhal numbers higher than 0.2 , noise dominates as shown by the flattening of the convective ridge. Along the serrations, milder changes are observed in the spectrum, confirming the small modification of the flow from the root to the tip, and implying no significant variation in the mean convection velocity of the structures over the serrations.

Finally, the spanwise correlation length of the surface pressure fluctuations is shown in Fig. 16. A small increase of $l_{z}$ is observed along the serration with larger correlation length in the low frequency range, in agreement with previous studies (Avallone et al. 2016). The observed behaviour

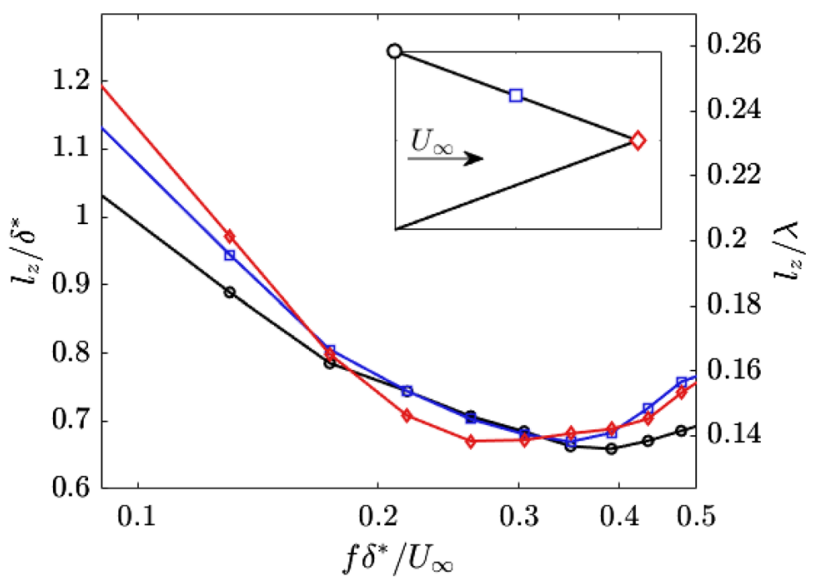

Fig. 16 Correlation length variation along the serration edge. Axes limits are given by the average inter-particle distance and resolved frequency is attributed to the deflection of the larger structures in the spanwise direction. At higher frequencies, a small increase in the structure length is captured at the very edge of the serration. This is possibly caused by the formation of additional structures over the edge and follows the level increase observed in the spectrum.

\section{Conclusions}

Large-scale time-resolved 3D-PIV measurements were carried out using Lagrangian particle tracking and helium-filled soap bubbles as flow tracers. VIC+ was used to reconstruct a dense velocity field and the pressure field was obtained by solving the Poisson equation. Measurements with a Pitot tube and with surface microphones were presented as validation for the technique, demonstrating the capability of the latter to correctly retrieve the flow information within a volume in the order of $10^{3} \mathrm{~cm}^{3}$.

Spectral analyses of the pressure fluctuations were possible up to $\mathrm{St}_{\delta^{*}}=1.3$, while spatial-temporal correlation measurements are accurate up to $\mathrm{St}_{\delta^{*}}=0.4$. The limitations suggest that constraints are still imposed over the particle density necessary to capture smaller structures and not related to the spatial discretization of the field reconstruction. Comparisons have shown that the technique applied is capable of correctly predict the boundary-layer flow above the viscous sublayer within the uncertainties expected.

The same technique is then extended to a serrated trailing-edge case, where the flow in both sides of the model is assessed through a thin Plexiglas wall. Result points out to a mild but consistent influence of the trailing-edge serrations over the flow features, similar to the wide serration experiment from Moreau and Doolan (2013). Overall, 
measurements of the pressure fluctuations around the serration edges point to the correct description of the flow with the measurement technique applied. The development of the wake around the serration gap is captured and seen to influence the edge pressure spectrum at mid-to-high frequencies. Variations of the correlation length are also observed close to the tip. It is found that the spanwise correlation length increases in the low-frequency range as also reported in the literature.

This study proves that the adopted techniques are suitable for aeroacoustics studies of large serrations at higher Reynolds numbers, correctly retrieving the spatial and temporal correlation of the surface pressure fluctuations.

Acknowledgements This study is supported by the SMARTANSWER project (Smart Mitigation of flow-induced Acoustic Radiation and Transmission for reduced Aircraft, surface traNSport, Workplaces and wind enERgy noise) which has received funding from the European Union's Horizon 2020 research and innovation program under the Marie Skłodowska-Curie grant agreement No. 722401. More information can be found on https://www.h2020-smartanswer.eu. The authors would also like to acknowledge Javed Mohd and Giuseppe Carlo Alp Caridi for conducting the measurements.

Open Access This article is licensed under a Creative Commons Attribution 4.0 International License, which permits use, sharing, adaptation, distribution and reproduction in any medium or format, as long as you give appropriate credit to the original author(s) and the source, provide a link to the Creative Commons licence, and indicate if changes were made. The images or other third party material in this article are included in the article's Creative Commons licence, unless indicated otherwise in a credit line to the material. If material is not included in the article's Creative Commons licence and your intended use is not permitted by statutory regulation or exceeds the permitted use, you will need to obtain permission directly from the copyright holder. To view a copy of this licence, visit http://creativecommons.org/licenses/by/4.0/.

\section{References}

Amiet RK (1976) Noise due to turbulent flow past a trailing edge. J Sound Vib 47:387-393. https://doi.org/10.1016/0022460X(76)90948-2

Avallone F, Pröbsting S, Ragni D (2016) Three-dimensional flow field over a trailing-edge serration and implications on broadband noise. Phys Fluids. https://doi.org/10.1063/1.4966633

Avallone F, van der Velden WCP, Ragni D, Casalino D (2018) Noise reduction mechanisms of sawtooth and combed-sawtooth trailing-edge serrations. J Fluid Mech 848:560-591. https://doi. org/10.1017/jfm.2018.377

Ayton LJ (2018) Analytic solution for aerodynamic noise generated by plates with spanwise-varying trailing edges. J Fluid Mech 849:448-466. https://doi.org/10.1017/jfm.2018.431

Bailey SCC, Hultmark M, Monty JP et al (2013) Obtaining accurate mean velocity measurements in high Reynolds number turbulent boundary layers using Pitot tubes. J Fluid Mech 715:642-670. https://doi.org/10.1017/jfm.2012.538

Bosbach J, Kühn M, Wagner C (2009) Large scale particle image velocimetry with helium filled soap bubbles. Exp Fluids 46:539_ 547. https://doi.org/10.1007/s00348-008-0579-0
Chase DM (1987) The character of the turbulent wall pressure spectrum at subconvective wavenumbers and a suggested comprehensive model. J Sound Vib 112:125-147. https://doi.org/10.1016/ S0022-460X(87)80098-6

Chong TP, Vathylakis A (2015) On the aeroacoustic and flow structures developed on a flat plate with a serrated sawtooth trailing edge. J Sound Vib 354:65-90. https://doi.org/10.1016/j.jsv.2015.05.019

Clauser FH (1956) The turbulent boundary layer. Adv Appl Mech 4:1-51. https://doi.org/10.1016/S0065-2156(08)70370-3

Corcos GM (1964) The structure of the turbulent pressure field in boundary-layer flows. J Fluid Mech 18:353-375

Faleiros DE, Tuinstra M, Sciacchitano A, Scarano F (2018) Heliumfilled soap bubbles tracing fidelity in wall-bounded turbulence. Exp Fluids 59:1-13. https://doi.org/10.1007/s00348-018-2502-7

Ghaemi S, Scarano F (2013) Turbulent structure of high-amplitude pressure peaks within the turbulent boundary layer. J Fluid Mech 735:381-426. https://doi.org/10.1017/jfm.2013.501

Ghaemi S, Ragni D, Scarano F (2012) PIV-based pressure fluctuations in the turbulent boundary layer. Exp Fluids 53:1823-1840. https ://doi.org/10.1007/s00348-012-1391-4

Goody M (2008) Empirical spectral model of surface pressure fluctuations. AIAA J 42:1788-1794. https://doi.org/10.2514/1.9433

Gruber M, Joseph P, Chong T (2011) On the mechanisms of serrated airfoil trailing edge noise reduction. In: 17th AIAA/CEAS aeroacoustics conf (32nd AIAA Aeroacoustics Conf 5-8. https://doi. org/10.2514/6.2011-2781

Gurka R, Liberzon A, Hefetz D et al (1999) Computation of pressure distribution using PIV velocity data. J Tribol 106:1-6. https://doi. org/10.1115/1.1631009

Howe MS (1978) A review of the theory of trailing edge noise. J Sound Vib 61:437-465. https://doi.org/10.1016/0022-460X(78)90391-7

Howe MS (1991a) Noise produced by a sawtooth trailing edge. J Acoust Soc Am 90:482-487. https://doi.org/10.1121/1.401273

Howe MS (1991b) Aerodynamic noise of a serrated trailing edge. J Fluids Struct 5:33-45. https://doi.org/10.1016/0889-9746(91)80010 $-B$

Howe MS (1998) Acoustics of fluid-structure interactions. Cambridge University Press, Cambridge

Hwang YF, Bonness WK, Hambric SA (2009) Comparison of semiempirical models for turbulent boundary layer wall pressure spectra. J Sound Vib 319:199-217. https://doi.org/10.1016/j. jsv.2008.06.002

Johansson AV, Her J-Y, Haritonidis JH (2006) On the generation of high-amplitude wall-pressure peaks in turbulent boundary layers and spots. J Fluid Mech 175:119. https://doi.org/10.1017/S0022 112087000326

Jones LE, Sandberg RD (2012) Acoustic and hydrodynamic analysis of the flow around an aerofoil with trailing-edge serrations. J Fluid Mech 706:295-322. https://doi.org/10.1017/jfm.2012.254

Lynch K, Scarano F (2013) A high-order time-accurate interrogation method for time-resolved PIV. Meas Sci Technol. https://doi. org/10.1088/0957-0233/24/3/035305

Lyu B, Azarpeyvand M, Sinayoko S (2016) Prediction of noise from serrated trailing edges. J Fluid Mech 793:556-588. https://doi. org/10.1017/jfm.2016.132

Moreau DJ, Doolan CJ (2013) Noise-reduction mechanism of a flatplate serrated trailing edge. AIAA J 51:2513-2522. https://doi. org/10.2514/1.j052436

Oerlemans S, Sijtsma P, Méndez López B (2007) Location and quantification of noise sources on a wind turbine. J Sound Vib 299:869883. https://doi.org/10.1016/j.jsv.2006.07.032

Palumbo D (2012) Determining correlation and coherence lengths in turbulent boundary layer flight data. J Sound Vib 331:3721-3737. https://doi.org/10.1016/j.jsv.2012.03.015

Pröbsting S, Scarano F, Bernardini M, Pirozzoli S (2013) On the estimation of wall pressure coherence using time-resolved 
tomographic PIV. Exp Fluids 54:1567. https://doi.org/10.1007/ s00348-013-1567-6

Pröbsting S, Tuinstra M, Scarano F (2015) Trailing edge noise estimation by tomographic particle image velocimetry. J Sound Vib 346:117-138. https://doi.org/10.1016/j.jsv.2015.02.018

Raffel M, Willert CE, Scarano F et al (2018) Particle image velocimetry. Springer, Berlin

Ragni D, Avallone F, van der Velden WCP, Casalino D (2019) Measurements of near-wall pressure fluctuations for trailing-edge serrations and slits. Exp Fluids 60:6. https://doi.org/10.1007/s0034 8-018-2654-5

Rodríguez-López E, Bruce PJK, Buxton ORH (2016) On the formation mechanisms of artificially generated high reynolds number turbulent boundary layers. Bound Layer Meteorol 160:201-224. https://doi.org/10.1007/s10546-016-0139-8

Roger M, Moreau S (2005) Back-scattering correction and further extensions of Amiet's trailing-edge noise model. Part 1: theory. J Sound Vib 286:477-506. https://doi.org/10.1016/j. jsv.2004.10.054

Romano GP (1995) Analysis of two-point velocity measurements in near-wall flows. Exp Fluids 20:68-83. https://doi.org/10.1007/ BF00189296

Sanders MPJ, De Santana LD, Azarpeyvand M, Venner CH (2018) Unsteady surface pressure measurements on trailing edge serrations based on digital MEMS microphones. AIAA/CEAS Aeroacoust Conf 2018:1-12. https://doi.org/10.2514/6.2018-3290

Sanjosé M, Moreau S, Lyu B, Ayton LJ (2019) Analytical, numerical and experimental investigation of trailing-edge noise reduction on a Controlled Diffusion airfoil with serrations. In: 25th AIAA/ CEAS Aeroacoustics Conf. https://doi.org/10.2514/6.2019-2450
Scarano F, Ghaemi S, Caridi GCA et al (2015) On the use of heliumfilled soap bubbles for large-scale tomographic PIV in wind tunnel experiments. Exp Fluids. https://doi.org/10.1007/s0034 8-015-1909-7

Schanz D, Gesemann S, Schröder A (2016) Shake-The-Box: Lagrangian particle tracking at high particle image densities. Exp Fluids 57:1-27. https://doi.org/10.1007/s00348-016-2157-1

Schanz D, Geisler R, Voss C et al (2018) Large scale volumetric flow measurements in a turbulent boundary layer. In: Proceedings 18th international symposium on flow visualization

Schneiders JFG, Scarano F (2016) Dense velocity reconstruction from tomographic PTV with material derivatives. Exp Fluids 57:1-22. https://doi.org/10.1007/s00348-016-2225-6

van Oudheusden BW (2013) PIV-based pressure measurement. Meas Sci Technol. https://doi.org/10.1088/0957-0233/24/3/032001

Violato D, Moore P, Scarano F (2011) Lagrangian and Eulerian pressure field evaluation of rod-airfoil flow from time-resolved tomographic PIV. Exp Fluids 50:1057-1070. https://doi.org/10.1007/ s00348-010-1011-0

Wieneke B (2008) Volume self-calibration for 3D particle image velocimetry. Exp Fluids 45:549-556. https://doi.org/10.1007/ s00348-008-0521-5

Publisher's Note Springer Nature remains neutral with regard to jurisdictional claims in published maps and institutional affiliations. 